\title{
A Study of the Finite Sample Properties of EMM, GMM, QMLE, and MLE for a Square-Root Interest Rate Diffusion Model
}

\author{
Hao Zhou ${ }^{1}$ \\ Mail Stop 91 \\ Federal Reserve Board \\ Washington, DC 20551 \\ First Draft: April 1997 \\ Last Revised: August 2000
}

\begin{abstract}
${ }^{1}$ I would like to thank George Tauchen for his valuable advice and Ronald Gallant for his insightful comments. The views expressed in this paper reflect those of the author and do not represent those of the Board of Governors of the Federal Reserve System or other members of its staff. I am grateful to an anonymous referee for his or her constructive suggestions for making the study complete. This paper has benefited from discussions with Matthew Pritsker, Christopher Downing, Mark Coppejans, Chien-Te Hsu, and the participants of the Duke financial economics seminar. Please contact Hao Zhou with questions and comments: Trading Risk Analysis Section, Division of Research and Statistics, Federal Reserve Board, Washington DC 20551 USA; Phone 1-202-452-3360; Fax 1-202-452-3819; e-mail hao.zhou@frb.gov. An earlier draft of the paper was distributed under the title "Finite Sample Properties of Efficient Method of Moments and Maximum Likelihood Estimations for a Square-Root Diffusion Process".
\end{abstract}




\begin{abstract}
This paper performs a Monte Carlo study on Efficient Method of Moments (EMM), Generalized Method of Moments (GMM), Quasi-Maximum Likelihood Estimation (QMLE), and Maximum Likelihood Estimation (MLE) for a continuous-time square-root model under two challenging scenarios - high persistence in mean and strong conditional volatility - that are commonly found in estimating the interest rate process. MLE turns out to be the most efficient of the four methods, but its finite sample inference and convergence rate suffer severely from approximating the likelihood function, especially in the scenario of highly persistent mean. QMLE comes second in terms of estimation efficiency, but it is the most reliable in generating inferences. GMM with lag-augmented moments has overall the lowest estimation efficiency, possibly due to the ad hoc choice of moment conditions. EMM shows an accelerated convergence rate in the high volatility scenario, while its overrejection bias in the mean persistence scenario is unacceptably large. Finally, under a stylized alternative model of the US interest rates, the overidentification test of EMM obtains the ultimate power for detecting misspecification, while the GMM J-test is increasingly biased downward in finite samples.
\end{abstract}

Keywords: Monte Carlo Study, Efficient Method of Moments, Maximum Likelihood Estimation, Square-Root Diffusion, Quasi-Maximum Likelihood, Generalized Method of Moments.

JEL classification: C15; C22; C52 


\section{Introduction}

When estimating a continuous time model in finance, one often faces the difficulty of partial observability. Usually the continuous time record is not available, since the data is discretely sampled. A further complication is that the transitional density of the stochastic process does not always have a closed-form solution. Due to the lack of a tractable likelihood function, much of the interest among researchers has turned to nonlikelihood-based approaches. For instance, by exploiting the analytical solutions of the first two moments, the Quasi-Maximum Likelihood Estimation (QMLE), as discussed in Bollerslev and Wooldridge (1992) conveniently circumvents the need to evaluate density function, although the asymptotic validity of QMLE imposes certain restrictions on the innovation density (Newey and Steigerwald 1997). Moreover, the Generalized Method of Moments (GMM) by Hansen (1982) and Hansen and Scheinkman (1995) further reduces the reliance on distribution assumptions by matching the empirical moments with the theoretical ones. Meanwhile, the Simulated Method of Moments (SMM) in time-series application (Ingram and Lee 1991, Duffie and Singleton 1993) minimizes the reliance on distribution assumptions by matching the empirical moments with the simulated ones. Both GMM and SMM are robust to the misspecification of likelihood functions, while retaining a parametric model to conduct simulation or projection. However, these methods of moments suffer from the ad hoc choice of moment conditions and must presume the existence of arbitrary population moments; and the chisquare specification test of the overidentifying restrictions is subject to severe overrejection bias (Hansen, Heaton, and Yaron 1996, Andersen and Sørenson 1996). Furthermore, the efficiency loss of parameter estimates is closely related to the high cost in estimating the weighting matrix, as the variance-covariance matrix is typically heteroskedastic and serially correlated (Andersen and Sørenson 1996). The Wald test is also found to exceed its asymptotic size due to the difficulty in estimating the residue spectral-density matrix (Burnside and Eichenbaum 1996).

The Efficient Method of Moments (EMM), introduced by Bansal, Gallant, Hussey, and Tauchen (1995) and Gallant and Tauchen (1996c), endogenously selects the moment con-

ditions during the procedure's first step. A seminonparametric score generator (SNP) uses the Fourier-Hermite polynomial to approximate the underlying transitional density. As an 
orthogonal series estimator, the SNP density has a fast uniform convergence, given the smoothness of the underlying distribution function. A suitable model selection criterion, e.g., the Schwarz's Bayesian Information Criterion (BIC), is used to choose the direction and complexity of the auxiliary model expansion. The second stage of EMM is simply an SMM-type estimator, minimizing the quasi-maximum likelihood score functions that are chosen appropriately in the first stage. Since the score functions are orthogonal, the weighting matrix (i.e., the information matrix from the quasi-maximum likelihood estimator) should be nearly serially uncorrelated. Hence the asymptotic variance estimator approaches the minimum bound, and the parameter estimates are asymptotically as efficient as MLE. It is proven that for an ergodic stochastic system with partially observed data, the efficiency of EMM approaches that of MLE, as the number of moment conditions and the number of lags entering each moment increase with the sample size (Gallant and Long 1997). Another salient feature of EMM is the capability to detect a misspecified structural model, if the auxiliary model is rich enough such that Hermite polynomial scores approximate the true scores fairly well (Tauchen 1997). Under correct specification of the maintained model, the normalized objective function value converges in distribution to a chi-square distribution. Under misspecification, the unnormalized objective function converges almost surely to a constant. For the particular choice of a score generator, this constant may be zero and the chi-square test has little power against the alternatives. If the data generating process is adequately captured by a more flexible nonparametric score generator, the constant is positive and rejection of misspecification is almost certain.

Recent Monte Carlo studies documented significant efficiency gains of EMM over GMM (Andersen, Chung, and Sørenson 1999), but with similar overrejection problems in specification tests (Chumacero 1997). In more analytical fashion, Gallant and Tauchen (1998a) show that EMM outperforms the conventional method of moments (CMM) for a representative class of econometric models, given the same number of moments being selected. However, there is no universal theory regarding the efficiency of EMM versus that of CMM, and the comparison must be made case-by-case (Gallant and Tauchen 1998a). Therefore choosing EMM over QMLE, as in Dai and Singleton (2000), should be accompanied by solid argument or Monte Carlo evidence. This paper complements these comparative studies in several areas. First, the Monte Carlo setup is a continuous time model, like many recent applications 
of EMM, which have focused on the stochastic differential equations. Second, I consider the relative efficiency of EMM with respect to asymptotically efficient MLE, computationally efficient QMLE, and empirically attractive GMM. Third, both blindfold and educated choice of moment conditions are examined, so as to best imitate the realistic approaches taken by researchers using EMM. Thus, the main contribution of this paper is to provide Monte Carlo evidence which shows that when the analytical density or moments are unavailable, EMM performs reasonably well compared to the infeasible MLE, QMLE, or GMM. Further, under two challenging scenarios - mean persistence and volatility cluster, each method has its own strength and weakness, in terms of estimation efficiency, parameter inference, and specification test.

A square-root diffusion process (Cox, Ingersoll, and Ross 1985) is chosen as the vehicle for conducting the Monte Carlo study. On the one hand, the CIR model is simple enough to give closed-form solutions for both the transitional density and the asset pricing formula. On the other hand, it is rich enough to generate a highly persistent volatility and nonGaussian error distribution. The square-root process seems to be a good starting point to model more complicated financial time series data. EMM estimation of the interest rate diffusions is reported by Gallant and Tauchen (1998b), and the square-root model is firmly rejected. With a closed-form transitional density, the dynamic maximum likelihood estimation was implemented for the two-factor CIR model (Pearson and Sun 1994, Duffie and Singleton 1997). Gibbons and Ramaswamy (1993) employed a GMM estimator, using the stochastic Euler equations to generate the moment conditions. Their results favor the squareroot model. The most recent interest in affine term structure (Dai and Singleton 2000) can be viewed as an immediate extension of the multifactor square-root model. The CIR model also has an explicit marginal density in terms of the drift and volatility functions, which motivated a nonparametric specification test (Aït-Sahalia 1996b). Conley, Hansen, Luttmer, and Scheinkman (1997) implemented a GMM estimator for a subset of the parameters by exploiting the reversibility of a stationary Markov chain. Fisher and Gilles (1996) proposed a general QMLE estimator for the CIR type diffusion processes.

The remaining sections are organized as following: Section 2 discusses some properties of the square-root model and characterizes the implementations of MLE, QMLE, and GMM; Section 3 introduces the relatively new Efficient Method of Moments estimator; Section 4 
designs the Monte Carlo experiment and suggests the benchmark model choice; Section 5 reports the major findings; and Section 6 concludes.

\section{Square-Root Model and Parametric Estimator}

This section defines a maximum likelihood estimator for the square-root model, based on a Poisson-mixing-Gamma characterization of the likelihood function. A quasi-maximum likelihood estimator is also available with analytical solutions to the first two conditional moments. Augmenting these two moments with instrumental variables gives a generalized method of moments estimator found in the literature.

\subsection{Probabilistic Solution to Square-Root Model}

It is a well-known result that the square-root model,

$$
d r_{t}=\left(a_{0}+a_{1} r_{t}\right) d t+b_{0} r_{t}^{1 / 2} d W_{t}
$$

satisfies the regularity conditions for both a strong solution (pathwise convergent) and a weak solution (convergent in probability) (Karatzas and Shreve 1997). A strong solution obviously implies a weak one, but not vice versa. If (1) $a_{0}>0$, (2) $b_{0}>0$, (3) $a_{1}<0$, and (4) $b_{0}^{2} \leq 2 a_{0}$, then the square-root model has a unique fundamental solution (Feller 1951). The marginal density is a Gamma distribution, and the transitional density is a type I Bessel function distribution or a noncentral chi-square distribution with a fractional order (Cox et al. 1985). Intuitively, condition (3) gives mean reversion, and condition (4) ensures the stationarity.

The marginal Gamma distribution is

$$
f\left(r_{0} \mid \nu, \omega\right)=\frac{\omega^{\nu}}{\Gamma(\nu)} r_{0}^{\nu-1} e^{-\omega r_{0}},
$$

where $\nu=2 a_{0} / b_{0}^{2}, \omega=-2 a_{1} / b_{0}^{2}$, and $\Gamma(\cdot)$ is the Gamma function. ${ }^{1}$ The unconditional mean and variance are

$$
\begin{aligned}
& E\left(r_{0}\right)=\frac{-a_{0}}{a_{1}}=\frac{\nu}{\omega}, \\
& V\left(r_{0}\right)=\frac{b_{0}^{2} a_{0}}{2 a_{1}^{2}}=\frac{\nu}{\omega^{2}} .
\end{aligned}
$$

\footnotetext{
${ }^{1}$ In some textbooks and software, the second parameter of the Gamma density is written in terms of $1 / \omega$ instead of $\omega$ (Johnson and Kotz 1970).
} 
Notice that the first two moments merely identify the marginal distribution. Higher order moments are simply nonlinear functions of the first two moments. The marginal density alone can not identify all three parameters in the diffusion process. Any GMM-type estimator must add at least one lagged instrumental variable (Gibbons and Ramaswamy 1993). A rejection of the marginal distribution can reject the square-root model; however, a non-rejection does not provide enough information for judging a particular parameter setting (Aït-Sahalia 1996b). Transitional information must be exploited to fully identify the dynamic structure.

${ }^{2}$ The conditional density is

$$
\begin{aligned}
f\left(r_{1} \mid r_{0} ; a_{0}, a_{1}, b_{0}\right) & =c e^{-u-v}\left(\frac{v}{u}\right)^{\frac{q}{2}} I_{q}\left(2(u v)^{\frac{1}{2}}\right), \\
q & =\frac{2 a_{0}}{b_{0}^{2}}-1
\end{aligned}
$$

where $c=-2 a_{1} /\left(b_{0}^{2}\left(1-e^{a_{1}}\right)\right), u=c r_{0} e^{a_{1}}$, and $v=c r_{1} . I_{q}(\cdot)$ is a modified Bessel function of the first kind with a fractional order $q$ (Oliver 1972). The conditional mean and variance are

$$
\begin{aligned}
E\left(r_{1} \mid r_{0}\right) & =r_{0} e^{a_{1}}-\frac{a_{0}}{a_{1}}\left(1-e^{a_{1}}\right), \\
V\left(r_{1} \mid r_{0}\right) & =r_{0}\left(-\frac{b_{0}^{2}}{a_{1}}\right) e^{a_{1}}\left(1-e^{a_{1}}\right)+\frac{b_{0}^{2} a_{0}}{2 a_{1}^{2}}\left(1-e^{a_{1}}\right)^{2} .
\end{aligned}
$$

According to the stationary property, the limit of the transitional density as the time interval goes to infinity, is exactly the marginal density. Therefore any estimation strategy or specification test that exploits the transitional density will naturally nest the ones that rely on the marginal density.

It is a common practice in the literature to call this distribution a "noncentral chisquare distribution." However, the "integer order noncentral chi-square distribution" does not naturally extend to the "fractional order noncentral chi-square distribution." The latter arises commonly from the solution to a diffusion process (Feller 1971), while the former arises from the sample standard deviation of independent, nonidentical, noncentered, normal random variables (Johnson and Kotz 1970).

\footnotetext{
${ }^{2}$ The process is sampled with weekly observations and the time interval of a week is normalized to be one.
} 


\subsection{Analytical Foundations for MLE, QMLE, and GMM}

In industry and academics alike, one popular method in estimating the square-root model for interest rates is the Discretized Maximum Likelihood Estimation (DMLE), i.e., a misspecified QMLE based on the time discretization of the conditional mean $E\left(r_{1} \mid r_{0}\right) \approx a_{0}+\left(1+a_{1}\right) r_{0}$ and variance $V\left(r_{1} \mid r_{0}\right) \approx b_{0}^{2} r_{0}$. As pointed out by Lo (1988), DMLE is generally not consistent. The parameter estimates are asymptotically biased, since both moments are misspecified.

When implementing MLE for the square-root model, the Bessel function representation of the likelihood function is not at all a convenient form (Pearson and Sun 1994, Duffie and Singleton 1997). An alternative Poisson-mixing-Gamma characterization can be inferred from the simulation strategy suggested by Devroye (1986). Within the admissible parameter region, one can substitute the Bessel function with an infinite series expansion (Oliver 1972). With appropriate transformations $(y=v, \lambda=q+1$, and $\beta=\sqrt{2 u})$, the alternative mixing formula comes out nicely,

$$
\begin{aligned}
f(y) & =\sum_{j=0}^{\infty} \frac{y^{j+\lambda-1} e^{-y}}{\Gamma(j+\lambda)} \cdot \frac{\left(\frac{\beta^{2}}{2}\right)^{j} e^{-\frac{\beta^{2}}{2}}}{j !} \\
& =\sum_{j=0}^{\infty} \operatorname{Gamma}(y \mid j+\lambda, 1) \cdot \operatorname{Poisson}\left(j \mid \frac{\beta^{2}}{2}\right) .
\end{aligned}
$$

One needs to be cautious that the Poisson weights are not constant, but rather condition on the previous realization $r_{0}$. This formula corresponds to the "Poisson driven Gamma process" in Feller (1971). The only difference is that $\lambda=q+1$ remains a fractional number, not an integer. The evaluation of the log-likelihood function in MLE is greatly simplified when using the Poisson-mixing-Gamma formula. It is fairly easy to achieve the single precision $10^{-8}$ by truncating the Poisson distribution around 100. One can also avoid any complication of complex value or non-convergence in evaluating the Bessel function. ${ }^{3}$

The exact expressions for the conditional mean and variance (equations 7 and 8) suggest

\footnotetext{
${ }^{3}$ This mixing approach to likelihood function works well for the high volatility case, but the approach fails for the mean persistence case, because the latter is close to the unit root or nonstationary region (see Section 4.1 for details on choosing the benchmark scenario) and because the evaluation of the likelihood function easily diverges. To implement MLE in the mean persistence case, I adopt the asymptotic expansion formula (Oliver 1972) in the same fashion as Pearson and Sun (1994).
} 
a quasi-maximum likelihood estimator (QMLE) for the square-root model,

$$
\max _{\left\{a_{0}, a_{1}, b_{0}\right\}} \sum_{t=1}^{T-1} \log \frac{1}{\sqrt{2 \pi V\left(r_{t+1} \mid r_{t}\right)}} \exp \left\{-\frac{\left[r_{t+1}-E\left(r_{t+1} \mid r_{t}\right)\right]^{2}}{2 V\left(r_{t+1} \mid r_{t}\right)}\right\} .
$$

QMLE is shown to be root- $n$ consistent (Bollerslev and Wooldridge 1992) and asymptotically normal (Newey and Steigerwald 1997) under some mild regularity conditions.

For comparison purposes, a generalized method of moments (GMM) estimator can be constructed from the following moment vector

$$
f_{t}\left(a_{0}, a_{1}, b_{0}\right)=\left[\begin{array}{c}
r_{t+1}-E\left(r_{t+1} \mid r_{t}\right) \\
r_{t}\left[r_{t+1}-E\left(r_{t+1} \mid r_{t}\right)\right] \\
V\left(r_{t+1} \mid r_{t}\right)-\left[r_{t+1}-E\left(r_{t+1} \mid r_{t}\right)\right]^{2} \\
r_{t}\left\{V\left(r_{t+1} \mid r_{t}\right)-\left[r_{t+1}-E\left(r_{t+1} \mid r_{t}\right)\right]^{2}\right\}
\end{array}\right]
$$

The parameter is estimated by $\min g_{T}^{\prime} W g_{T}$, where $g_{T}=1 / T \sum_{t=1}^{T-1} f_{t}$ and $W$ is the asymptotic variance-covariance matrix of $g_{T}$ (Hansen 1982). A two step estimator is adopted here.

\section{$2.3 \quad$ Inference}

Since we know the true parameter value for MLE, the likelihood ratio tests can determine how often the confidence region centered at the estimated parameter value contains the truth. The same principle may be extended to QMLE if the expected quasi-likelihood function is uniquely maximized at the truth (satisfying identification requirement). In addition to the correct specification of the mean and variance, the remaining innovation error must be centered at zero or have a symmetric distribution (Newey and Steigerwald 1997). QMLE for the square-root model meets these conditions. Also, the sample-size normalized criterion function value in GMM provides a specification test distributed as a Chi-square (1).

The standard error for an individual parameter is estimated by the inverted Hessian formula in MLE, the OPG-Hessian-OPG formula in QMLE, and the Gradient-Weighting Matrix-Gradient formula in GMM.

\subsection{Simulation}

This characterization (equation 9) also defines a composite method to simulate the squareroot process (Devroye 1986). First, one draws a random number $j$ from the Poisson $\left(j \mid \beta^{2} / 2\right)$. 
Then, one draws another random number $y$ from the $\operatorname{Gamma}(y \mid j+\lambda, 1)$. Finally, one calculates the desired state variable $r_{1}$ by $r_{1}=y / c$. Notice that the realized $r_{1}$ is the conditioning value $r_{0}$ in the next draw. The initial value $r_{0}$, when starting a simulation run, can be set to the theoretical unconditional mean. To pass on the transient effect, the first 1000 realizations can be discarded.

\section{Efficient Method of Moments Estimation}

This section describes the EMM estimator in a univariate case. For formal discussions, see Gallant and Tauchen (1996c), Tauchen (1997), and Gallant and Long (1997).

\subsection{Approximating True Density with Auxiliary Model}

Denote the invariant probability measure implied by the underlying data generating process as the $p$-model. It is assumed that the direct maximum likelihood estimation of the $p$-model is not available. However, any smooth density function can be approximated arbitrarily close by a Hermite polynomial expansion.

Consider a scalar case. Let $y$ be the random variable, $x$ be the lagged $y$, and $\theta$ be the parameter. The auxiliary $f$-model has a density function defined by a modified Hermite polynomial,

$$
f(y \mid x, \theta)=C\left\{[\mathcal{P}(z, x)]^{2} \phi\left(y \mid \mu_{x}, \sigma_{x}^{2}\right)\right\},
$$

where $\mathcal{P}$ is a polynomial with degree $K_{z}$ in $z$ and thus the square of $\mathcal{P}$ makes the density positive. The argument of the polynomial is $z$, which is the transformation $z=\left(y-\mu_{x}\right) / \sigma_{x}$. The coefficient of the polynomial is another polynomial of degree $K_{x}$ in $x$. The constant in the polynomial is set to 1 for identification. $C$ is a normalizing factor to make the density proper. ${ }^{4} \phi(\cdot)$ is a normal density of $y$ with conditional mean $\mu_{x}$ and conditional variance $\sigma_{x}^{2}$. The length of the auxiliary model parameter is determined by the lag in mean $L \mu$, lag in variance $L_{r}$, lag in polynomial coefficient $L_{p}$, polynomial degree $K_{z}$, and polynomial degree $K_{x}$. Let $\left\{\tilde{y}_{t}\right\}_{t=1}^{n}$ be the observed data and $\tilde{x}_{t-1}$ be the lagged observations. The sample mean

\footnotetext{
${ }^{4}$ In the case of multivariate density, the interaction terms in both the Hermite polynomial and the coefficient polynomial can be set to zero, in order for a gradual expansion of the auxiliary model. See above references for details.
} 
log-likelihood function is defined by

$$
\mathcal{L}_{n}\left(\theta,\left\{\tilde{y}_{t}\right\}_{t=1}^{n}\right)=\frac{1}{n} \sum_{t=1}^{n} \log \left[f\left(\tilde{y}_{t} \mid \tilde{x}_{t-1}, \theta\right)\right] .
$$

A quasi-maximum-likelihood estimator is obtained by

$$
\tilde{\theta}=\arg \max _{\theta} \mathcal{L}_{n}\left(\theta,\left\{\tilde{y}_{t}\right\}_{t=1}^{n}\right)
$$

The dimension of the auxiliary $f$-model, the length of $\theta$, is selected by Schwarz's Bayesian Information Criterion (BIC). There are different choices of information criteria for optimal model selection. For a finite dimensional stationary process, BIC with a larger penalty for model complexity proves to be consistent, while the Akaike's Information Criterion (AIC) will overfit the model. On the other hand, if the true dimension is infinity or increases to infinity with the sample size, AIC with a smaller penalty for model complexity is optimal (Zheng and Loh 1995). The dimensions of the $f$-model needs to be as large as the $p$-model to meet the identification condition. ${ }^{5}$

\subsection{Matching Auxiliary Scores with Minimum Chi-Square}

From the first-stage seminonparametric estimates, one obtains the fitted scores as the moment conditions,

$$
m_{n}(\tilde{\theta})=\frac{1}{n} \sum_{t=1}^{n} \frac{\partial}{\partial \theta} \log f\left(\tilde{y}_{t} \mid \tilde{x}_{t-1}, \tilde{\theta}\right) .
$$

In the second stage, a SMM-type estimator is implemented in the following way. Although the direct MLE for $p$-model is assumed to be impossible, the simulation from the structural model (e.g., the stochastic differential equation) is readily available. Let $\left\{\hat{y}_{t}\right\}_{t=1}^{N}$ be a long simulation from a candidate value of $\rho$, the parameter of the maintained structural model. The auxiliary score functions can be reevaluated by numerical integration of of the score functions with the simulated data,

$$
\hat{m}_{N}(\rho, \tilde{\theta})=\frac{1}{N} \sum_{t=1}^{N} \frac{\partial}{\partial \theta} \log f\left(\hat{y}_{t} \mid \hat{x}_{t-1}, \tilde{\theta}\right),
$$

\footnotetext{
${ }^{5}$ Even when the underlying $p$-model has a fixed dimension (i.e., a fixed number of parameters), the auxiliary $f$-model can still have an increasing order in finite sample sizes, because of the approximation from auxiliary model to true model.
} 
and the minimum chi-square estimator is simply,

$$
\hat{\rho}=\arg \min _{\rho}\left\{\hat{m}_{N}(\rho, \tilde{\theta})^{\prime} \tilde{\mathcal{I}}^{-1} \hat{m}_{N}(\rho, \tilde{\theta})\right\}
$$

where the weighting matrix $\tilde{\mathcal{I}}^{-1}$ is estimated by the mean-outer-product of scores from the auxiliary model

$$
\tilde{\mathcal{I}}=\frac{1}{n} \sum_{t=1}^{n}\left[\frac{\partial}{\partial \theta} \log f\left(\tilde{y}_{t} \mid \tilde{x}_{t-1}, \tilde{\theta}\right)\right]\left[\frac{\partial}{\partial \theta} \log f\left(\tilde{y}_{t} \mid \tilde{x}_{t-1}, \tilde{\theta}\right)\right]^{\prime} .
$$

Remember that the finite sample efficiency loss of the GMM-type estimators is largely attributed to the high cost and low accuracy in estimating the serially correlated weighting matrix. In EMM the moment conditions (score functions) of the first step QMLE are orthogonal by construction; hence the information matrix is diagonal or nearly diagonal (i.e., serially uncorrelated). EMM will be asymptotically as efficient as MLE if the following conditions are met: the dimension of the auxiliary model is sufficiently large $(K \rightarrow \infty)$, the lag in the the auxiliary model is sufficiently long $(L \rightarrow \infty)$, and the simulation from the maintained structural model is sufficiently long $(N \rightarrow \infty)$. This encompasses both Markovian and non-Markovian cases (Gallant and Long 1997).

\subsection{Overrejection and Misspecification}

The normalized criterion function value in the EMM estimation,

$$
c_{n}^{2}=n \hat{m}_{N}(\hat{\rho}, \tilde{\theta})^{\prime} \tilde{\mathcal{I}}^{-1} \hat{m}_{N}(\hat{\rho}, \tilde{\theta}),
$$

forms a specification test for the overidentifying restrictions. Under the correct specification

of the maintained model (Tauchen 1997), we have $c_{n}^{2} \stackrel{\mathcal{D}}{\longrightarrow} \mathcal{X}^{2}\left(l_{\theta}-l_{p}\right)$, where the degree of freedom equals the parameter length of the auxiliary model minus that of the structural model. However, if the maintained model is misspecified (Tauchen 1997), we have

$$
\frac{c_{n}^{2}}{n} \stackrel{a . s .}{\longrightarrow} \bar{c}^{2}=\hat{m}_{N}(\bar{\rho}, \bar{\theta})^{\prime} \overline{\mathcal{I}}^{-1} \hat{m}_{N}(\bar{\rho}, \bar{\theta})>0,
$$

where $\bar{\theta}, \bar{\rho}$, and $\overline{\mathcal{I}}$ are the asymptotic pseudo-true values under the maintained misspecification. As long as the sample size $n$ is large enough, the SNP score generator will be rich enough such that the false passage $\bar{c}^{2}=0$ will not occur under misspecification. 


\section{Monte Carlo Design and Benchmark Choice}

Technical aspects of the Monte Carlo experiment are summarized here. Most importantly, two demanding scenarios - mean persistence and high volatility - are chosen as the benchmarks. Also, a maintained model of interest rate with stochastic volatility and level feedback is designated as the data generating process for comparing the power of detecting misspecification.

\subsection{Experimental Design}

All computations are performed on Sun Unix Workstations-Sparc10, Sparc20, and Pentium II-300. Programs for generating random samples and GMM, QMLE, and MLE estimations of the square-root model are written in FORTRAN language. The FORTRAN codes for SNP and EMM are modified from SNP Version 8.5 (Gallant and Tauchen 1996b) and EMM Version 1.3 (Gallant and Tauchen 1996a), incorporating automatic SNP search by BIC in some of the EMM estimations. NPSOL (Gill, Murray, Saunders, and Wright 1991) is the optimization routine used for all of the programs.

The number of Monte Carlo replications is chosen as 1000 for each scenario and each estimator. Two finite sample sizes - 500 and 1500 weekly observations - are used for contrasting the asymptotic behavior of each estimator. To generate the pseudo-random samples, the Poisson-mixing-Gamma formula in Section 2 is implemented, and the 1000 initial stretch is discarded each time in order to pass on the transient effect. The second stage of EMM estimation is similar to SMM. The simulation size should be at least 30,000 . EMM does stabilize from 50,000 to 75,000 and could have more Monte Carlo errors at 100,000. Thus 50,000 turns out to be a conservative but economical choice (Gallant and Tauchen 1998b).

To choose a SNP score generator in the EMM estimation, one can either let BIC automatically decide the SNP dimension in each replication, or, one can use a "posterior" fixed SNP specification. For the comparisons with MLE, QMLE, and GMM estimators, I use the fixed SNP score in EMM estimation, such that the overrejection test statistics has the same degree of freedom for each sample size. However, in order to choose an appropriate fixed SNP score, I first run EMM 1000 times with the automatic SNP score generator. I then adopt one particular score for the 500 sample size and another for the 1500 sample 
size. These specifications are at relatively higher dimensions, with abundant occurrence but without severe rejection. A by-product of the EMM estimation with automatic SNP score is that some light can be shed on how the overrejection bias varies with the number of moments chosen by the SNP estimation. ${ }^{6}$

In terms of the computing time, each QMLE run takes about 3-5 seconds; each GMM run takes about 5-10 seconds; each MLE run takes about 5-10 minutes; and each EMM run takes about 1-2 hours. Note that MLE requires numerical approximation to the likelihood function and also that EMM uses numerical integration to evaluate the moment conditions. EMM estimator is programmed up and ready to be implemented (Gallant and Tauchen 1996b, Gallant and Tauchen 1996a). One only needs to incorporate the square-root model in the simulation code. The developing time for QMLE and EMM is minimal, while GMM especially MLE requires a lot of fine-tuning. Overall, EMM is still the most computationally intensive method, although there are numerical techniques to speed up the estimation (e.g., parallel processing or antithetic simulation).

\subsection{Benchmark Model}

To select a suitable parameter setting, we start with the empirical result from Gallant and Tauchen $(1998 \mathrm{~b}), d r_{t}=\left(0.02491-0.00285 r_{t}\right) d t+0.0275 r_{t}^{1 / 2} d W_{t}$. Using equations 3,4 , and 6-8, one can calculate the unconditional mean and variance, the Bessel function order, and the conditional mean and variance. It is not difficult to see that this original specification, Scenario 1 in Table 1 , features low mean-reversion $\left(E\left(r_{t+1} \mid r_{t}\right)\right.$ is nearly the unit-root) and low conditional volatility $\left(V\left(r_{t+1} \mid r_{t}\right)\right.$ is close to zero). Also the unconditional variance is unusually small, representing an abnormally quiet process. The order of the Bessel function, twice of which corresponds to the degree of freedom for an integer order noncentral chi-square distribution, is so large that the conditional density looks almost Gaussian. Not withstanding all these short comings, scenario 1 is still the typical empirical result. It poses an important challenge to researchers in fitting the highly persistent, nearly unit-root conditional mean process, although its volatility structure and innovation density are not rich enough. Hence

\footnotetext{
${ }^{6}$ This automatic-plus-fixed SNP-EMM procedure mimics the realistic situation, when an empirical researcher not only relies on BIC as an objective criterion in model selection but also incorporates prior subjective information to expand the auxiliary model-e.g, the E-GARCH score generator in Andersen et al. (1999).
} 
it will only rarely generate the high order ARCH or the high degree polynomial in the SNP score. Based on the empirical results in Gallant and Tauchen (1998b), I use the established SNP score for Scenario 1-s14140 for the 500 sample size and s14141 for the 1500 sample $\operatorname{size}^{7}$ - and I report only the fixed score EMM estimations. The Scenario 1 in Table 1 is termed an LMR-LCV specification (low-mean-reversion and low-conditional-volatility).

If one increases only the variance parameter $b_{0}$ from Scenario 1 to $2-4$ in Table 1 , the Bessel function order $q$ decreases gradually from the Gaussian-like specification, but the conditional volatility is still negligible. Alternatively, one can increase both the mean parameters $a_{0}$ and $a_{1}$ by a factor of 100 and the variance parameter $b_{0}$ by a factor of 10 from Scenario 1 to 5 as in Table 1, while holding the unconditional mean and variance constant. This change will increase the conditional volatility slightly, but the Bessel function order $q$ is still quite large (resembling a Gaussian-like distribution). If one increases the variance parameter $b_{0}$ from Scenario 5 to 6-8 as shown in Table 1, both high conditional volatility and small Bessel function order are achieved. Scenario 8 is rich enough in both the conditional volatility and non-Gaussian innovation, hence Scenario 8 is suitable for examining the automatic SNP score EMM estimations. Strong volatility cluster is another challenge in fitting the short interest rate, although the high persistence in mean is sacrificed somewhat. Scenario 8 in Table $1, d r_{t}=\left(2.491-0.285 r_{t}\right) d t+1.1 r_{t}^{1 / 2} d W_{t}$, is termed an HMR-HCV specification (high-mean-reversion and high-conditional-volatility).

One should not arbitrarily relate either Scenario 1 or Scenario 8 alone to the real interest rate, but put together they represent the most important features in short rate process. There is a fundamental concern with how flexible the square-root model can be. In order to fit the real interest rate data, one would like to hold the unconditional mean $\left(-a_{0} / a_{1}\right)$ constant without explosion $\left(b_{0} \leq 4 a_{0}^{2}\right)$, resembling a stationary interest rate process. In addition one would like to achieve high persistence in both conditional mean and variance. With only three parameters to manipulate, it seems impossible to satisfy all four constraints simultaneously. This indicates that the square-root model may not be flexible enough to model the interest rate dynamics. To find a more suitable model requires a fourth degree of

\footnotetext{
${ }^{7}$ s14140 means 1 lag in mean, 4 lag in variance, 1 lag in coefficient polynomial, 4 degree Hermite polynomial, and 0 degree coefficient polynomial. s14141 only differs in 1 lag coefficient polynomial. There is more detailed discussion of the SNP structure in Section 3.1.
} 
freedom, for example, a stochastic volatility component (Gallant and Tauchen 1998b).

\subsection{Testing Misspecification}

The goal is to find a true data generating process, of which an adequate auxiliary score will not accommodate a misspecified model. As discussed in Section 1, the square-root model is widely used in fitting the short rate process, but most serious studies have rejected this specification. So it is natural to adopt the square-root process as a misspecified model and use a non-rejected model as the true data generating process (for the short interest rate). A recent study by Gallant and Tauchen (1998b) gave the most favorable evidence for the following specification,

$$
\begin{aligned}
& d r_{t}=\left(0.014-0.002 r_{t}\right) d t+\left(0.043-0.018 r_{t}\right) e^{u_{t}} d W_{1 t} \\
& d u_{t}=\left(-0.006 r_{t}-0.157 u_{t}\right) d t+\left(0.593-0.052 u_{t}\right) d W_{2 t}
\end{aligned}
$$

The short rate process $r_{t}$ has a linear drift and a linear diffusion, with the diffusion multiplied by an unobserved (exponential) stochastic volatility term $e^{u_{t}}$. The short rate $r_{t}$ is only partially observable in discrete time. The latent stochastic volatility process $u_{t}$ also has a linear drift and a linear diffusion, with the drift including a short rate level feedback. This model adequately passed the specification test for various simulation sizes and greatly outperformed the competing models in terms of reprojecting the conditional density and the conditional volatility. It would be a very suitable choice for the true data generating process.

When we fit the misspecified square-root model $d r_{t}=\left(a_{0}+a_{1} r_{t}\right) d t+b_{0} r_{t}^{1 / 2} d W_{t}$ to the interest rate data simulated from the maintained true specification (equations 20 and 21), the drift is correctly specified as a linear function, and the misspecification comes only into the diffusion. From Itô's formula we know that the conditional mean is also correctly specified as linear; therefore the drift parameters are consistent estimates (Aït-Sahalia 1996a). It is equivalent to the case where Ordinary Least Square is consistent, but unaccounted heteroskedastic and/or correlated error structure may cause very noisy and inefficient estimates. The misspecified diffusion generates inconsistent estimates of the conditional variance, which may cause serious distortions in pricing discount bond yields or other interest rate sensitive derivatives. Therefore detecting the misspecification in diffusion or volatility is a critical challenge to researchers and practitioners. 


\section{Monte Carlo Results}

Tables 2 through 6 and Figures 1 through 9 summarize the major findings of this paper. The discussions are organized along topics, and extensive comparisons are made across QMLE, EMM, GMM, and MLE. The main focuses are finite sample efficiency, overrejection bias of EMM under the null, and the detection of maintained misspecification in EMM and GMM. Both the automatic score generator and the fixed score generator are used in EMM. The likelihood ratio tests in MLE and QMLE provide joint inferences under the null.

\subsection{Simulation Schemes}

The Poisson-mixing-Gamma formula is a useful characterization of the transitional density of the square-root model. The simulation accuracy based on the distribution function can provide an independent check for the derivations in Section 2. The Monte Carlo study is not alone in needing a reliable simulator; further applications of MLE with this formula also require some justification. A cornerstone of the EMM estimator - a simulation-based estimator - is the discretized approximation to stochastic differential equations. EMM uses a weak-order 2 scheme (Kloeden and Platen 1992). To assess these simulation approaches, some empirical statistics from long realizations $(100,000)$ are compared to their theoretical counterparts.

Table 2 lists the calculation of two moments and three quantiles. Clearly both schemes from exact distribution and time discretization work reasonably well. In both persistent mean and strong volatility cases, the simulated moments and quantiles are very close to the model implied ones. It is not surprising that the probabilistic method is slightly better than the discretized method, although the difference is negligible.

\subsection{Score Generator}

An important feature of EMM is the endogenous moment selection by a seminonparametric score generator (SNP), which contrasts with the ad hoc choice of moment conditions in some less sophisticated GMM or SMM estimators. The optimal SNP search and the inexpensive weighting matrix estimate are key to the efficiency argument, and hopefully they also improve the overrejection test. It is worthwhile to check whether the SNP score captures the 
distribution features of different dependent structures before launching the full-scale Monte Carlo experiment. Tables 3 (500 sample size) and Table 4 (1500 sample size) report the SNP searches for the 8 scenarios in Table 1. For each setting, the frequencies of all kinds of model choices among 100 replications are listed. Model dimension is represented by a five-digit number, which stands for, consecutively, lag in mean, lag in variance, lag in polynomial, degree of Hermite polynomial, and degree of Hermite coefficient polynomial.

Scenario 1 in Tables 3 and 4 is the LMR-LCV case (low-mean-reversion, low-conditionalvolatility). Not surprisingly the Gaussian auto-regression specification of 10100 dominates other choices. This finding is consistent with the fact that the true density is nearly Gaussian under this parameter setting (see Table 1). Moving from Scenario 1 to 2, 3, and 4, the conditional volatility increases gradually, since the variance parameter $b_{0}$ is altered (see Table 1). The dominating choice is still Gaussian, and the chances of ARCH and/or non-Gaussian specifications increase slightly. Moving toward Scenarios 5-8, both mean parameters $a_{0}$ and $a_{1}$ as well as variance parameter $b_{0}$ are altered (see Table 1), and ultimately one reaches the HMR-HCV case (high-mean-reversion, high-conditional-volatility). It is clear that the SNP search favors the nonlinear, nonparametric AR-ARCH specification. This is consistent with the low Bessel function order and high conditional variance (Table 1). Largely due to this "distribution-dependent" or "data-dependent" score generator, the EMM estimator is claimed to be asymptotically efficient and hopefully more reliable in the specification test.

Also evident from Tables 3 and 4 is that larger sample sizes enable the SNP to pick up higher model dimensions. In fact, the asymptotic efficiency argument requires that the number of moment conditions and the lags entering each moment increase with the sample size (Gallant and Long 1997).

A salient question is whether the structural model can be identified when the SNP search does pick the Gaussian-AR(1) score. This corresponds to a quasi-maximum likelihood estimator based on the innovation assumption

$$
z_{t}=\left(r_{t}-\alpha_{0}-\alpha_{1} r_{t-1}\right) / \alpha_{2} \sim N(0,1)
$$

Since the conditional mean is correctly specified, the QMLE of $\alpha_{0}$ and $\alpha_{1}$ is a consistent estimator of $e^{a_{1}}$ and $-a_{0} / a_{1}\left(1-e^{a_{1}}\right)$. It is just an Ordinary Least Square with a heteroskedastic and serially correlated error term (Aït-Sahalia 1996a). The conditional variance is mis- 
specified as the constant $\alpha_{2}$. However, according to the theory of misspecified maximum likelihood estimation (White 1994), the estimator $\hat{\alpha}_{2}$ may converge to a pseudo-true value $\bar{\alpha}_{2}$. The key argument is that the misspecified asymptotic variance $\bar{\alpha}_{2}$ must be a function of the true variance parameter $b_{0}$, since both conditional variance and unconditional variance are determined by $b_{0}$. These asymptotic relations, two explicit and one implicit, are indeed the binding functions in the language of Indirect Inference (Gourierous, Monfort, and Renault 1993). Obviously the structural parameters $a_{0}, a_{1}$, and $b_{0}$ are exactly identified by the auxiliary parameters $\alpha_{0}, \alpha_{1}$, and $\alpha_{2}$. EMM is thus a feasible first-order approximation toward the Indirect Inference (Gallant and Long 1997).

\subsection{Estimation Bias and Finite Sample Efficiency}

Table 5 (persistent mean case ) and Table 6 (strong volatility case) report the mean bias, medium bias, and root-mean-squared error (RMSE) across MLE, QMLE, GMM, and EMM between 500 and 1500 sample sizes.

First look at the case of persistent mean (Table 5). The biases are very large for the drift estimates $\left(a_{0}\right.$ and $\left.a_{1}\right)$ but quite small for the diffusion parameter $\left(b_{0}\right)$. The biases reduce with the sample size, except for MLE and GMM. MLE is the most efficient in achieving the smallest RMSE; however, the drift parameter estimates diverge-RMSE does not shrink with the sample size. ${ }^{8}$ EMM seems to be more efficient than GMM but less efficient than QMLE. The diffusion parameter estimation in GMM does not converge, similar to the drift parameter in MLE. The drift parameter estimates in QMLE and the intercept of drift in GMM seem to converge faster than root- $n$. The convergence rate of EMM in these finite samples is very close to $\sqrt{3}$. Overall the drift estimates are more biased and noisy than the diffusion estimate, and the close-to-unit-root mean persistence causes some unusual convergence problems in finite samples. The rank in order of efficiency from highest to lowest would be MLE, QMLE, EMM, GMM, as expected.

In the case of strong volatility (Table 6), all parameter estimates have very small biases (relative to parameter value), and all biases shrink appropriately as the sample size increases.

\footnotetext{
${ }^{8}$ When the square-root is close to the non-stationary region, the mixing formula for approximating the likelihood easily diverges in MLE estimations. One has to rely on the asymptotic expansion which introduces the asymptotic biases. It should be pointed out that the simulation scheme based on the mixing formula is still sound, since the parameter is fixed in simulations.
} 
In terms of the efficiency, the RMSE's of MLE, QMLE, and GMM are shrinking approximately at the rate $\sqrt{3}$, but the RMSE of EMM decreases faster than root- $n$. Recall that EMM should be asymptotically as efficient as MLE, as the auxiliary SNP score generator adopts an increasing dimension with the sample size. Overall MLE achieves the highest efficiency, QMLE comes second at $\mathrm{T}=500$ and third at $\mathrm{T}=1500, \mathrm{EMM}$ is third at $\mathrm{T}=500$ and second at $\mathrm{T}=1500$, GMM is best for the intercept parameter of drift but is worst for the slope parameter of drift and the diffusion parameter. The finding that some parameter estimates in GMM and QMLE are slightly more efficient than MLE, can be attributed to the fact that "exact" likelihood function in MLE needs to be numerically approximated while the moment conditions in GMM or QMLE are in closed forms.

\subsection{Parameter Inference}

The finite sample distributions of the standardized t-test statistics for individual parameters are summarized in Figure 1 (persistent mean) and Figure 2 (strong volatility). Standard Gaussian kernel smoothing is adopted here. QMLE works well in both cases; EMM is reliable in the latter case, while MLE and GMM seem not to perform in either case.

In the case of mean persistence (Figure 1), MLE seems to have asymptotic biases for the drift parameter (underestimating $a_{0}$ and overestimating $a_{1}$ ), but the diffusion parameter is perfectly approximated by its asymptotic distribution. QMLE works equally well for the diffusion parameter, while its estimates for the drift parameters, though having some finite sample bias ( $a_{0}$ has upward bias and $a_{1}$ has downward bias), are dissipating asymptotically. GMM is not biased in estimating the drift, but the standard error is too small (high peak in the middle) and the estimation variation is too large (fat tails on both sides). Its inference for the diffusion is scattering everywhere. EMM suffers from high variations for all the parameters.

The case of strong volatility (Figure 2) looks much different. MLE has almost negligible biases, but it still understates the standard error and produces fat tails. QMLE works perfectly well, especially in the tails where the 1\%,5\%, and 10\% t-tests are usually conducted. GMM suffers heavily from both understating and overstating the standard errors. EMM comes close to QMLE, and its bias and variation are shrinking with the increasing sample sizes. 
Several factors may contribute to the unusual t-test distributions: (1) high persistence in the mean makes the simulated data look like a unit-root and the MLE, GMM, and EMM estimators can not distinguish it from a persistent yet stationary situation in finite samples; (2) the so-called "exact" likelihood in MLE is approximated by its series expansion (in strong volatility case) or its asymptotic expansion (in persistent mean case); (3) the t-test is a Wald test and lacks invariance to nonlinear transformations. ${ }^{9}$

\subsection{Overrejection Bias and Number of Moments}

The Monte Carlo results on EMM with automatic SNP score generator can reveal some connections between the number of overidentifying moments and the overrejection rate. Figures 3 and 4 report this experiment for Scenario 8- the case of strong volatility-which can generate richer SNP scores. "Number of Overidentified Moments" refers to the difference between the number of moments chosen automatically by BIC (which varies each trial) and the number of structural parameters in the square-root model (which is fixed at 3). The occurrence curve is the percentage of how many times BIC chooses a particular number of moments over the 1000 Monte Carlo replications. Further, the rejection curves (fixed at the $5 \%$ level) tell you the percentage of how many times EMM rejects the null square-root specification at this particular "Number of Overidentified Moments" over the number of times that BIC picks this particular SNP score. ${ }^{10}$

The asymptotic size of the specification test is fixed at $5 \%$. The occurrence rates show the frequencies of different numbers of moment conditions in 1000 replications. On average, the $5 \%$ gross overrejection rate in automatic score $\mathrm{EMM}$ is about $20 \%$ for $\mathrm{T}=500$ and about $25 \%$ for $\mathrm{T}=1500$. Some important features need to be mentioned. First, the rejection curve does not uniformly shoot up when more moment conditions are included, since these moments are optimally selected by the SNP score generator. Second, the rejection rates are more stable at $\mathrm{T}=1500$ than $\mathrm{T}=500$, as more moments and lags are included. Third, the rejection rate could be remarkably small for certain low dimensions as well as for some high dimensions. Since BIC tends to underfit the auxiliary model in small samples, the

\footnotetext{
${ }^{9}$ For example, the drift function in the square-root model $a_{0}+a_{1} r_{t}$ can be reparameterized as $\kappa\left(\theta-r_{t}\right)$, then $\theta$ becomes a nonlinear transformation of $a_{0}$ and $a_{1}$.

${ }^{10}$ Figures 7 and 8 are the same, except that the data is simulated from the alternative stochastic volatility process while the EMM is carried out for a misspecified square-root process.
} 
higher level unrejected score is more likely capturing the true distribution. If the lower level unrejected score did select the true specification, the rejection rate is likely to shoot up beyond that level. The implication for empirical work is that an SNP search should go beyond the first optimal choice by BIC.

\subsection{Specification Test}

Based on the insights gathered from the EMM experiments with automatic SNP score generator, I choose some "educated" fixed SNP scores for the strong volatility case. For the 500 sample size I use s10111 (1 lag in mean, 0 lag in variance, 1 lag in polynomial coefficient, 1 degree in Hermite polynomial, and 1 degree in coefficient polynomial), and for the 1500 sample I use s10121 (1 lag in mean, 0 lag in variance, 1 lag in polynomial coefficient, 2 degrees in Hermite polynomial, and 1 degree in coefficient polynomial). In the case of mean persistence, which is widely estimated in empirical studies, I use the established SNP scores $-\mathrm{s} 14140$ for $\mathrm{T}=500$ and $\mathrm{s} 14141$ for $\mathrm{T}=1500$. In Figures 5 and 6 , these EMM J-tests results are contrasted with GMM. With a knowledge of the true parameters, one can also perform a likelihood ratio test in MLE or a quasi-likelihood ratio test in QMLE to see whether the confidence ball concentrated at the estimated parameter contains the true parameter as often as suggested by the chi-square (3) random variable. This approach is not available to the empirical researcher, since no true parameter is known. However, in the Monte Carlo setting, one can use these "infeasible" tests to judge the reliability of MLE or QMLE.

When testing the mean persistence scenario (Figure 5), QMLE gives the best inference as the overrejection bias is small and is reducing with increases in the sample size. GMM and EMM have severe overrejection biases, although the biases shrink rapidly in a large sample size. EMM seems to be worse than GMM, even with improved estimates of the weighting matrix. The MLE LR-test diverges as the sample size increases, which can be attributed to the asymptotic bias of parameter estimates introduced by approximating the likelihood function. Turning to the case of strong volatility (Figure 6), one can see that the QMLE LR-test and the EMM J-test have almost perfect size, and the overrejection bias is negligibly small. On the contrary, the MLE LR-test has severe underrejection, which does not shrink asymptotically. Even worse, the GMM J-test has a large underrejection bias, 
which is diverging with increases in sample sizes.

There are at least four sources of overrejection or underrejection bias in GMM-type estimators: inaccurate and costly estimates of the weighting matrix; unseasoned selection of the moment conditions; an inadequate number of moments to capture the distribution feature; and simply a small sample bias. A generic EMM approach overcomes the first two problems by adopting a serially uncorrelated information matrix and an optimal SNP score generator. The conservative BIC procedure in EMM may choose too few moments, but one can rectify this problem by using additional information and extending the SNP search beyond the BIC choice. The remaining small sample bias can be remedied by enlarging the sample size. The above arguments carry through here, except in the case of persistent mean, where the EMM estimator may mistake the data as coming from a unit-root process.

\subsection{Detecting Misspecification}

The power for detecting misspecification is examined in two aspects: (1) first, using automatic SNP score EMM procedure to study the power in relation to the number of moments, which is optimally chosen by BIC; (2) second, using a fixed SNP-score EMM procedure to compare the power with GMM, where EMM adopts an "educated" choice of the moment conditions while GMM adopts the simple lag-augmented moment conditions. ${ }^{11}$

In the first stage, the benchmark stochastic volatility model (equations 20 and 21 in Section 4.3) is used to simulate 1000 replications for the 500 and 1500 sample sizes, and then a square-root diffusion process is fitted to the data. This time I let BIC automatically choose the best SNP score generator. Since the drift is linear, the conditional mean with lag one is correctly specified. For the 500 sample size, $91 \%$ of the trials select lag 1; and for the 1500 sample size, $93 \%$ of the trials select lag 1 . The choice of conditional standard deviation is all over the place, due to the nature of nonlinear stochastic volatility. For $\mathrm{T}=$ 500 , the selection is scattered mainly from lag 1 to lag 4 , and for $\mathrm{T}=1500$, it is scattered mainly from lag 3 to lag 6 . The choices of $K_{z}$ and $K_{x}$ are predominantly zero. Figures 7 and

\footnotetext{
${ }^{11}$ One should be cautioned that the alternative model of interest rate process adopted here is only one special case, and a full scale study of misspecification issue is clearly outside the scope of this paper. Even in the following limited example, the performance of EMM is not unrelated with the established ARCH (Engle 1982) and GARCH (Bollerslev 1986) filtering of the stochastic volatility process. In fact, the power of EMM will be optimal if the first stage SNP auxiliary model - with an ARCH (earlier version) or GARCH (recent version) leading term-adequately captures the interest rate dynamics.
} 
8 plot the $5 \%$ rejection rates against the number of overidentified moments along with the occurrence rates of these moment choices. The highlight is that the probability of rejecting a misspecified model does converge to one very quickly. At $\mathrm{T}=500$, the $5 \%$ level rejection rate is around $80-90 \%$ for a range of overidentified moments between 1 to 6 , and beyond that the rejection rate is almost $100 \%$ (Figure 7 ). At $\mathrm{T}=1500$, the rejection rate is always close to $100 \%$, except in an exactly identified case (Figure 8).

In the second stage, similar to the study of the overrejection issue, I fix the SNP score generator and look at the rejection rate uniformly along the 1\%-100\% test level. The fixed SNP score generator for the 500 sample size is s13100 (1 lag in mean and 3 lags in variance), and the chi-square test has a degree of freedom that is 3 . When $\mathrm{T}=1500$, the score is

fixed at s15100 (1 lag in mean and 5 lags in variance), with 5 degrees of freedom. Figure 9 gives the rejection plot for the EMM J-test statistics, in comparison with GMM which has 1 overidentified moment. The upshot is that the EMM has the power to detect a misspecified model, and the power quickly converges to one as the sample size increases from 500 to 1500 . In contrast, the GMM has a serious underrejection problem for the maintained misspecification, and the underrejection bias becomes larger as sample size increases - ultimately loosing the power to detect misspecification. The explanation is quite simple: EMM chooses carefully a SNP moment generator by BIC, while standard GMM simply uses the lag-augmented instruments.

\section{Conclusions}

This paper performs a Monte Carlo study on Efficient Method of Moments (EMM), Generalized Method of Moments (GMM), Quasi-Maximum Likelihood Estimation (QMLE), and Maximum Likelihood Estimation (MLE) for a continuous-time square-root model under two challenging scenarios - high persistence in mean and strong conditional volatility - that are commonly encountered when estimating the empirical interest rate process.

MLE achieves the highest efficiency, while its inferences on individual parameters and overall specification are not very reliable and are even misleading on some occasions. QMLE is less efficient in comparison to MLE, but QMLE stands out as the best inference tool in both the individual t-test and overall LR-test. EMM shows a convergence rate faster than 
root- $n$, due to the expanding SNP score choice by BIC as sample size increases. EMM also provides better inference than GMM or MLE in a high volatility scenario. In the case of persistent mean - close to unit root in small samples - some asymptotics of MLE and GMM break down, as parameter estimates and test statistics diverge.

A number of lessons can be learned from this study: (1) MLE is not necessarily the best choice if the numerical approximation to the density is complex and/or the approximation tends to diverges near the non-stationary region; (2) QMLE is simple to implement and can be very reliable when the specification information is easily incorporated in the closed-form conditional mean and variance; (3) if there is no new information to be incorporated into the moment conditions, GMM can not be superior to QMLE; (4) when the true density or moment functions are not known, EMM is the only choice; its small sample performance is not necessarily inferior to the infeasible MLE or QMLE and is most likely superior to the infeasible GMM. 


\section{References}

Aït-Sahalia, Yacine (1996a), "Nonparametric Pricing of Interest Rate Derivatives," Econometrica, vol. 64, 527-560.

Aït-Sahalia, Yacine (1996b), "Testing Continuous-Time Models of the Spot Interest Rate," The Review of Financial Studies, vol. 9, 385-426.

Andersen, Torben G., Hyung-Jin Chung, and Bent E. Sørenson (1999), "Efficient Method of Moments Estimation of a Stochastic Volatility Model: A Monte Carlo Study," Journal of Econometrics, vol. 91, 61-87.

Andersen, Torben G. and Bent E. Sørenson (1996), "GMM Estimation of a Stochastic Volatility Model: A Monte Carlo Study," Journal of Business and Economic Statistics, vol. 14, $328-352$.

Bansal, Ravi, A. Ronald Gallant, Robert Hussey, and George Tauchen (1995), "Nonparametric Estimation of Structural Models for High-Frequency Currency Market Data," Journal of Econometrics, vol. 66, 251-287.

Bollerslev, Tim (1986), "Generalized Autoregressive Conditional Heteroscedasticity," Journal of Econometrics, vol. 31, 307-327.

Bollerslev, Tim and Jeffery Wooldridge (1992), "Quasi-Maximum Likelihood Estimators and Inference in Dynamic Models with Time-Varying Covariances," Econometric Review, vol. $11,143-172$.

Burnside, Craig and Martin Eichenbaum (1996), "Small-Sample Properties of GMM-Based Wald Tests," Journal of Business and Economic Statistics, vol. 14, 294-308.

Chumacero, Rómulo A. (1997), "Finite Sample Properties of the Efficient Method of Moments," Studies in Nonlinear Dynamics and Econometrics, vol. 2, 35-51.

Conley, Tim, Lars Peter Hansen, Erzo Luttmer, and Jose Scheinkman (1997), "Short Term Interest Rates as Subordinated Diffusions," Review of Financial Studies, vol. 10, 525578. 
Cox, John C., Jonathan E. Ingersoll, and Stephen A. Ross (1985), "A Theory of the Term Structure of Interest Rates," Econometrica, vol. 53, 385-407.

Dai, Qiang and Kenneth J. Singleton (2000), "Specification Analysis of Affine Term Structure Models," Journal of Finance, forthcoming.

Devroye, Luc (1986), Non-Uniform Random Variate Generation, Spinger-Verlag.

Duffie, Darrell and Kenneth Singleton (1993), "Simulated Moments Estimation of Markov Models of Asset Prices," Econometrica, vol. 61, 929-952.

Duffie, Darrell and Kenneth Singleton (1997), “An Econometric Model of the Term Structure of Interest-Rate Swap Yields," Journal of Finance, vol. 52, 1287-1321.

Engle, Robert F. (1982), "Autoregressive Conditional Heteroscedasticity with Estimates of the Variance of U.K. Inflation," Econometrica, vol. 50, 987-1008.

Feller, Wiliam (1951), "Two Singular Diffusion Problems," Annals of Mathematics, vol. 54, $173-182$.

Feller, Wiliam (1971), An Introduction to Probability Theory and Its Applications, vol. 2, John Wiley \& Sons, Inc., Princeton University, 2nd ed.

Fisher, Mark and Christian Gilles (1996), "Estimating Exponential Affine Models of the Term Structure," Working Paper.

Gallant, A. Ronald and Jonathan R. Long (1997), "Estimating Stochastic Differential Equations Efficiently by Minimum Chi-Square," Biometrika, vol. 84.

Gallant, A. Ronald and George Tauchen (1996a), User's Guide for EMM: A Program for Efficient Method of Moments Estimation, 1st ed.

Gallant, A. Ronald and George Tauchen (1996b), User's Guide for SNP: A Program for Nonparametric Time Series Analysis, 8th ed.

Gallant, A. Ronald and George Tauchen (1996c), "Which Moment to Match?" Econometric Theory, vol. 12, 657-681. 
Gallant, A. Ronald and George Tauchen (1998a), "The Relative Efficiency of Method of Moments Estimators," Working Paper.

Gallant, A. Ronald and George Tauchen (1998b), "Reprojecting Partially Observed Systems with Application to Interest Rate Diffusions," Journal of the American Statistical Association, vol. 93, 10-24.

Gibbons, Michael R. and Krishna Ramaswamy (1993), "A Test of the Cox, Ingersoll, and Ross Model of the Term Structure," Review of Financial Studies, vol. 6, 619-658.

Gill, Philip E., Walter Murray, Michael A. Saunders, and Margaret H. Wright (1991), "User's Guide for NPSOL (Version 4.06): A Fortran Package for Nonlinear Programming," Tech. rep., Stanford University.

Gourierous, C., A. Monfort, and E. Renault (1993), "Indirect Inference," Journal of Applied Econometrics, vol. 8, s85-s118.

Hansen, Lars Peter (1982), "Large Sample Properties of Generalized Method of Moments Estimators," Econometrica, vol. 50, 1029-1054.

Hansen, Lars Peter, John Heaton, and Amir Yaron (1996), "Finite-Sample Properties of Some Alternative GMM Estimators," Journal of Business and Economic Statistics, vol. $14,262-280$.

Hansen, Lars Peter and Jose Alexandre Scheinkman (1995), "Back to the Future: Generalized Moment Implications for Continuous Time Markov Process," Econometrica, vol. $63,767-804$.

Ingram, Beth F. and B. S. Lee (1991), "Simulation Estimation of Time Series Models," Journal of Econometrics, vol. 47, 197-205.

Johnson, Norman L. and Samuel Kotz (1970), Distributions in Statistics: Continuous Univariate Distributions, vol. 2, John Wiley \& Sons.

Karatzas, Ioannis and Steven E. Shreve (1997), Brownian Motion and Stochastic Calculus, Springer. 
Kloeden, Peter E. and Eckhard Platen (1992), Numerical Solution of Stochastic Differential Equations, Applications of Mathematics, Springer-Verlag.

Lo, Andrew W. (1988), "Maximum Likelihood Estimation of Generalized It'ô Process with Discretely Sampled Data," Econometric Theory, vol. 4, 231-247.

Newey, Whitney K. and Douglas G. Steigerwald (1997), "Asymptotic Bias for QuasiMaximum-Likelihood Estimators in Conditional Heteroscedasticity Models," Econometrica, vol. 65, 587-599.

Oliver, F. W. J. (1972), Handbook of Mathematical Functions with Formulas, Graphs, and Mathematical Tables, John Wiley \& Sons.

Pearson, Neil D. and Tong-Sheng Sun (1994), "Exploiting the Conditional Density in Estimating the Term Structure: An Application to the Cox, Ingersoll, and Ross Model," Journal of Finance, vol. 49, 1279-1304.

Tauchen, George (1997), "New Minimum Chi-Square Methods in Empirical Finance," in "Advances in Econometrics, Seventh World Congress," (edited by Kreps, D. and K. Wallis), Cambridge University Press, Cambridge UK.

White, Halbert (1994), Estimation, Inference, and Specification Analysis, Cambridge University Press, University of California, San Diego.

Zheng, Xiaodong and Wei-Yin Loh (1995), "Consistent Variable Selection in Linear Models," Journal of the American Statistical Association, vol. 90, 1029-1054. 
Table 1: Benchmark Model Choice

The square-root model is $d r_{t}=\left(a_{0}+a_{1} r_{t}\right) d t+b_{0} r_{t}^{1 / 2} d W_{t}$. Scenario 1 is taken from Gallant and Tauchen (1998b). In Scenarios 2-4, the variance parameter $b_{0}$ is increased by a factor of 2, 3, and 4 respectively. From Scenario 1 to Scenario 5, the mean parameters $a_{0}$ and $a_{1}$ are multiplied by 100 and the variance parameter $b_{0}$ is multiplied by 10 . From Scenario 5 to Scenarios 6-8, the variance parameter $b_{0}$ is increased by a factor of 2,3 , and 4 respectively. $E\left(r_{t}\right), V\left(r_{t}\right), q, E\left(r_{t+1} \mid r_{t}\right)$, and $V\left(r_{t+1} \mid r_{t}\right)$ are calculated using equations 3, 4, and 6-8.

\begin{tabular}{lrrrr}
\hline \hline & Scenario 1 & Scenario 2 & Scenario 3 & Scenario 4 \\
& $a_{0}=0.02491$ & $a_{0}=0.02491$ & $a_{0}=0.02491$ & $a_{0}=0.02491$ \\
& $a_{1}=-0.00285$ & $a_{1}=-0.00285$ & $a_{1}=-0.00285$ & $a_{1}=-0.00285$ \\
& $b_{0}=0.0275$ & $b_{0}=0.055$ & $b_{0}=0.0825$ & $b_{0}=0.11$ \\
\hline$E\left(r_{t}\right)$ & 8.74 & 8.74 & 8.74 & 8.74 \\
$V\left(r_{t}\right)$ & 1.16 & 4.64 & 10.44 & 18.55 \\
Bessel $q$ & 64.88 & 15.47 & 6.32 & 3.12 \\
$E\left(r_{t+1} \mid r_{t}\right)$ & $0.997 r_{t}+0.025$ & $0.997 r_{t}+0.025$ & $0.997 r_{t}+0.025$ & $0.997 r_{t}+0.025$ \\
$V\left(r_{t+1} \mid r_{t}\right)$ & $0.001 r_{t}+0.000$ & $0.003 r_{t}+0.000$ & $0.007 r_{t}+0.000$ & $0.012 r_{t}+0.000$ \\
\hline \hline & Scenario 5 & Scenario 6 & Scenario 7 & Scenario 8 \\
& $a_{0}=2.491$ & $a_{0}=2.491$ & $a_{0}=2.491$ & $a_{0}=2.491$ \\
& $a_{1}=-0.285$ & $a_{1}=-0.285$ & $a_{1}=-0.285$ & $a_{1}=-0.285$ \\
& $b_{0}=0.275$ & $b_{0}=0.55$ & $b_{0}=0.825$ & $b_{0}=1.1$ \\
\hline$E\left(r_{t}\right)$ & 8.74 & 8.74 & 8.74 & 8.74 \\
$V\left(r_{t}\right)$ & 1.16 & 4.64 & 10.44 & 18.55 \\
Bessel $q$ & 64.88 & 15.47 & 6.32 & 3.12 \\
$E\left(r_{t+1} \mid r_{t}\right)$ & $0.75 r_{t}+2.17$ & $0.75 r_{t}+2.17$ & $0.75 r_{t}+2.17$ & $0.75 r_{t}+2.17$ \\
$V\left(r_{t+1} \mid r_{t}\right)$ & $0.05 r_{t}+0.07$ & $0.20 r_{t}+0.29$ & $0.45 r_{t}+0.64$ & $0.79 r_{t}+1.14$ \\
\hline \hline
\end{tabular}


Table 2: Comparing Simulation Schemes (100,000 Length)

For the square-root model $d r_{t}=\left(a_{0}+a_{1} r_{t}\right) d t+b_{0} r_{t}^{1 / 2} d W_{t}$, the marginal distribution is a Gamma (equation 2), and the theoretical values are calculated accordingly. Simulation by distribution is based on the Poisson-mixing-Gamma formula (equation 11) and is subsequently implemented in both the Monte Carlo data generation and the Maximum Likelihood Estimation. Simulation by discretization is based on the weak-order 2 scheme (Gallant and Long 1997) and underscores the Efficient Method of Moments. Scenario 1 (LMR-LCV) is the Low-Mean-Reversion Low-Conditional-Variance benchmark, and Scenario 8 (HMRHCV) is the High-Mean-Reversion High-Conditional-Variance alternative. Both scenarios are defined in Table 1.

\begin{tabular}{lrrr}
\hline \hline Scenario 1 & $\begin{array}{r}\text { Simulated by } \\
\text { LMR-LCV }\end{array}$ & $\begin{array}{r}\text { Simulated by } \\
\text { Distribution }\end{array}$ & $\begin{array}{r}\text { Theoretical } \\
\text { Discretization }\end{array}$ \\
Value \\
\hline Vean & 8.72 & 8.79 & 8.74 \\
5\% Quantile & 1.18 & 1.09 & 1.16 \\
Median & 6.91 & 7.19 & 7.05 \\
95\% Quantile & 8.72 & 8.72 & 8.70 \\
\hline \hline Scenario 8 & 10.50 & 10.57 & 10.58 \\
HMR-HCV & Distributed by & Simulated by & Theoretical \\
\hline Mean & 8.75 & Discretization & Value \\
Variance & 18.34 & 18.22 & 8.74 \\
5\% Quantile & 3.04 & 3.03 & 18.55 \\
Median & 8.08 & 8.02 & 3.05 \\
95\% Quantile & 16.70 & 16.67 & 16.05 \\
\hline \hline
\end{tabular}


Table 3: SNP Search for the Sample Size of 500

(This note applies to both Table 3 and Table 4.) These results are from 100 replications of each scenario with the sample sizes of 500 and 1500. The information criterion used in moment selection is Schwarz's BIC. Scenarios 1-8 are the same as those in Table 1. Each model specification is characterized by a 5-digit number. Each digit consecutively stands for lag in mean, lag in variance, lag in polynomial, degree of Hermite polynomial, and degree of Hermite coefficient polynomial.

\begin{tabular}{rrrrrrrr}
\hline \hline Scenario & 1 & Scenario & 2 & Scenario & 3 & Scenario & 4 \\
\hline Model & $\%$ & Model & $\%$ & Model & $\%$ & Model & $\%$ \\
10100 & 95 & 10100 & 96 & 10100 & 92 & 10100 & 88 \\
11100 & 3 & 20100 & 3 & 10110 & 4 & 11100 & 7 \\
20100 & 1 & 10110 & 1 & 11100 & 2 & 10110 & 1 \\
21100 & 1 & & & 20100 & 2 & 10120 & 1 \\
& & & & & & 20100 & 1 \\
& & & & & & 21100 & 1 \\
& & & & & & 11111 & 1 \\
\hline Scenario & 5 & Scenario & 6 & Scenario & 7 & Scenario & 8 \\
\hline Model & $\%$ & Model & $\%$ & Model & $\%$ & Model & $\%$ \\
10100 & 90 & 10100 & 77 & 10100 & 46 & 10111 & 25 \\
10110 & 5 & 10110 & 12 & 10110 & 25 & 10100 & 18 \\
20100 & 3 & 11110 & 4 & 10111 & 8 & 10110 & 15 \\
11100 & 1 & 11100 & 3 & 10121 & 7 & 10121 & 11 \\
10120 & 1 & 10111 & 1 & 10120 & 6 & 11110 & 10 \\
& & 10120 & 1 & 11110 & 3 & 11120 & 6 \\
& & 12110 & 1 & 10130 & 1 & 10120 & 4 \\
& & 20100 & 1 & 11100 & 1 & 12120 & 3 \\
& & & & 11120 & 1 & 10131 & 2 \\
& & & & 11130 & 1 & 11130 & 2 \\
& & & & & & 11111 & 1 \\
& & & & & & 21120 & 1 \\
\hline \hline
\end{tabular}


Table 4: SNP Search for the Sample Size of 1500

\begin{tabular}{|c|c|c|c|c|c|c|c|}
\hline Scenario & 1 & Scenario & $\overline{22}$ & Scenario & 3 & Scenario & 4 \\
\hline Model & $\%$ & Model & $\%$ & Model & $\%$ & Model & $\%$ \\
\hline 10100 & 96 & 10100 & 96 & 10100 & 85 & 10100 & 57 \\
\hline 10110 & 2 & 11100 & 4 & 11100 & 11 & 11100 & 15 \\
\hline 20100 & 1 & & & 20100 & 2 & 12100 & 9 \\
\hline \multirow{12}{*}{11100} & 1 & & & 12100 & 1 & 13100 & 5 \\
\hline & & & & 13100 & 1 & 11111 & 2 \\
\hline & & & & & & 15100 & 2 \\
\hline & & & & & & 16100 & 2 \\
\hline & & & & & & 11121 & 1 \\
\hline & & & & & & 14100 & 1 \\
\hline & & & & & & 14110 & 1 \\
\hline & & & & & & 15110 & 1 \\
\hline & & & & & & 16110 & 1 \\
\hline & & & & & & 16111 & 1 \\
\hline & & & & & & 18100 & 1 \\
\hline & & & & & & 25100 & 1 \\
\hline Scenario & 5 & Scenario & 6 & Scenario & 7 & Scenario & 8 \\
\hline Model & $\%$ & Model & $\%$ & Model & $\%$ & Model & $\%$ \\
\hline 10100 & 89 & 10111 & 43 & 10111 & 41 & 12120 & 12 \\
\hline 10110 & 9 & 10100 & 30 & 10121 & 13 & 10131 & 11 \\
\hline 10120 & 1 & 10110 & 10 & 11110 & 11 & 10121 & 10 \\
\hline \multirow[t]{17}{*}{11110} & 1 & 10120 & 6 & 11111 & 8 & 11110 & 8 \\
\hline & & 10121 & 4 & 11121 & 8 & 10121 & 7 \\
\hline & & 11110 & 2 & 10131 & 7 & 11140 & 7 \\
\hline & & 12120 & 2 & 10111 & 5 & 10111 & 5 \\
\hline & & 11111 & 1 & 12120 & 3 & 11130 & 5 \\
\hline & & 20111 & 1 & 10120 & 2 & 13120 & 5 \\
\hline & & 20120 & 1 & 10141 & 1 & 10122 & 4 \\
\hline & & & & 12110 & 1 & 12130 & 4 \\
\hline & & & & & & 11111 & 1 \\
\hline & & & & & & 11141 & 1 \\
\hline & & & & & & 11150 & 1 \\
\hline & & & & & & 11160 & 1 \\
\hline & & & & & & 12110 & 1 \\
\hline & & & & & & 14120 & 1 \\
\hline & & & & & & 21120 & 1 \\
\hline & & & & & & 21140 & 1 \\
\hline & & & & & & 22120 & 1 \\
\hline
\end{tabular}


Table 5: Finite Sample Bias and Efficiency Comparison for Scenario 1

For each sample size, 1000 Monte Carlo replications are generated from the square-root process, and the model is estimated by MLE, QMLE, GMM, and EMM respectively. Scenario 1 is the Low-Mean-Reversion Low-Conditional-Variance (LMR-LCV) case. EMM estimation in this table uses a fixed SNP score generator.

\begin{tabular}{|c|c|c|c|c|c|c|}
\hline \multirow[b]{2}{*}{ True Value } & \multicolumn{2}{|c|}{ Mean Bias } & \multicolumn{2}{|c|}{ Median Bias } & \multicolumn{2}{|c|}{$\overline{\mathrm{RMSE}}$} \\
\hline & $\mathrm{T}=500$ & $\mathrm{~T}=1500$ & $\mathrm{~T}=500$ & $\mathrm{~T}=1500$ & $\mathrm{~T}=500$ & $\mathrm{~T}=1500$ \\
\hline & \multicolumn{6}{|c|}{ Maximum Likelihood Estimation } \\
\hline$a_{0}=0.02491$ & -0.0123 & -0.0130 & -0.0119 & -0.0126 & 0.0125 & 0.0131 \\
\hline$a_{1}=-0.00285$ & 0.0014 & 0.0015 & 0.0014 & 0.0014 & 0.0014 & 0.0015 \\
\hline \multirow{2}{*}{$b_{0}=0.02750$} & $-4.4 \mathrm{e}-5$ & $2.5 \mathrm{e}-6$ & $-4.6 \mathrm{e}-5$ & $2.1 \mathrm{e}-5$ & 0.0009 & 0.0005 \\
\hline & \multicolumn{6}{|c|}{ Quasi-Maximum Likelihood Estimation } \\
\hline$a_{0}=0.02491$ & 0.0994 & 0.0285 & 0.0803 & 0.0209 & 0.1343 & 0.0437 \\
\hline$a_{1}=-0.00285$ & -0.0113 & -0.0033 & -0.0091 & -0.0025 & 0.0153 & 0.0050 \\
\hline \multirow[t]{2}{*}{$b_{0}=0.02750$} & $3.0 \mathrm{e}-5$ & $1.2 \mathrm{e}-5$ & $4.1 \mathrm{e}-5$ & $1.9 \mathrm{e}-5$ & 0.0009 & 0.0005 \\
\hline & \multicolumn{6}{|c|}{ Generalized Method of Moments } \\
\hline$a_{0}=0.02491$ & 0.0019 & 0.0023 & $4.7 \mathrm{e}-5$ & $-5.1 \mathrm{e}-7$ & 0.2418 & 0.0960 \\
\hline$a_{1}=-0.00285$ & -0.0012 & 0.0022 & $-7.9 e-5$ & $-5.8 e-5$ & 0.0539 & 0.0481 \\
\hline \multirow[t]{2}{*}{$b_{0}=0.02750$} & 0.0040 & 0.0075 & $-7.3 e-6$ & $6.1 \mathrm{e}-6$ & 0.1256 & 0.1264 \\
\hline & \multicolumn{6}{|c|}{ Efficient Method of Moments } \\
\hline$a_{0}=0.02491$ & 0.0451 & 0.0407 & $2.6 \mathrm{e}-4$ & 0.0085 & 0.1252 & 0.0944 \\
\hline$a_{1}=-0.00285$ & -0.0054 & -0.0048 & $-8.1 e-5$ & -0.0012 & 0.0149 & 0.0112 \\
\hline$b_{0}=0.02750$ & -0.0015 & -0.0003 & $-4.8 \mathrm{e}-6$ & $-4.3 \mathrm{e}-7$ & 0.0076 & 0.0041 \\
\hline
\end{tabular}


Table 6: Finite Sample Bias and Efficiency Comparison for Scenario 8

For each sample size, 1000 Monte Carlo replications are generated from the square-root process, and the model is estimated by MLE, QMLE, GMM, and EMM respectively. Scenario 8 is the How-Mean-Reversion How-Conditional-Variance (HMR-HCV) case. EMM estimation in this table uses a fixed SNP score generator.

\begin{tabular}{ccccccc}
\hline \hline & \multicolumn{2}{c}{ Mean Bias } & \multicolumn{2}{c}{ Median Bias } & \multicolumn{3}{c}{ RMSE } \\
True Value & $\mathrm{T}=500$ & $\mathrm{~T}=1500$ & $\mathrm{~T}=500$ & $\mathrm{~T}=1500$ & $\mathrm{~T}=500$ & $\mathrm{~T}=1500$ \\
\hline \multicolumn{7}{c}{ Maximum Likelihood Estimation } \\
\hline$a_{0}=2.491$ & -0.0832 & -0.0663 & -0.0679 & -0.0524 & 0.1337 & 0.0923 \\
$a_{1}=-0.285$ & 0.0085 & 0.0058 & 0.0029 & 0.0010 & 0.0251 & 0.0161 \\
$b_{0}=1.100$ & 0.0024 & -0.0016 & 0.0060 & 0.0000 & 0.0432 & 0.0263 \\
\hline \multicolumn{7}{c}{ Quasi-Maximum Likelihood Estimation } \\
\hline$a_{0}=2.491$ & 0.0742 & 0.0224 & 0.0022 & 0.0006 & 0.3613 & 0.2111 \\
$a_{1}=-0.285$ & -0.0100 & -0.0020 & -0.0071 & -0.0011 & 0.0448 & 0.0258 \\
$b_{0}=1.100$ & 0.0023 & 0.0003 & 0.0015 & -0.0001 & 0.0430 & 0.0246 \\
\hline \multicolumn{7}{c}{ Generalized Method of Moments } \\
\hline$a_{0}=2.491$ & 0.0003 & 0.0039 & 0.0001 & 0.0001 & 0.1023 & 0.0541 \\
$a_{1}=-0.285$ & 0.0186 & 0.0110 & 0.0001 & 0.0005 & 0.0784 & 0.0561 \\
$b_{0}=1.100$ & 0.0097 & 0.0041 & -0.0023 & -0.0018 & 0.0838 & 0.0581 \\
\hline \multicolumn{7}{c}{ Efficient Method of Moments } \\
\hline$a_{0}=2.491$ & 0.1323 & -0.0067 & 0.0433 & -0.0173 & 0.4891 & 0.2000 \\
$a_{1}=-0.285$ & -0.0310 & -0.0022 & -0.0199 & -0.0000 & 0.0694 & 0.0257 \\
$b_{0}=1.100$ & -0.0218 & -0.0137 & -0.0091 & -0.0122 & 0.0618 & 0.0296 \\
\hline \hline
\end{tabular}


MLE: $a_{0}$
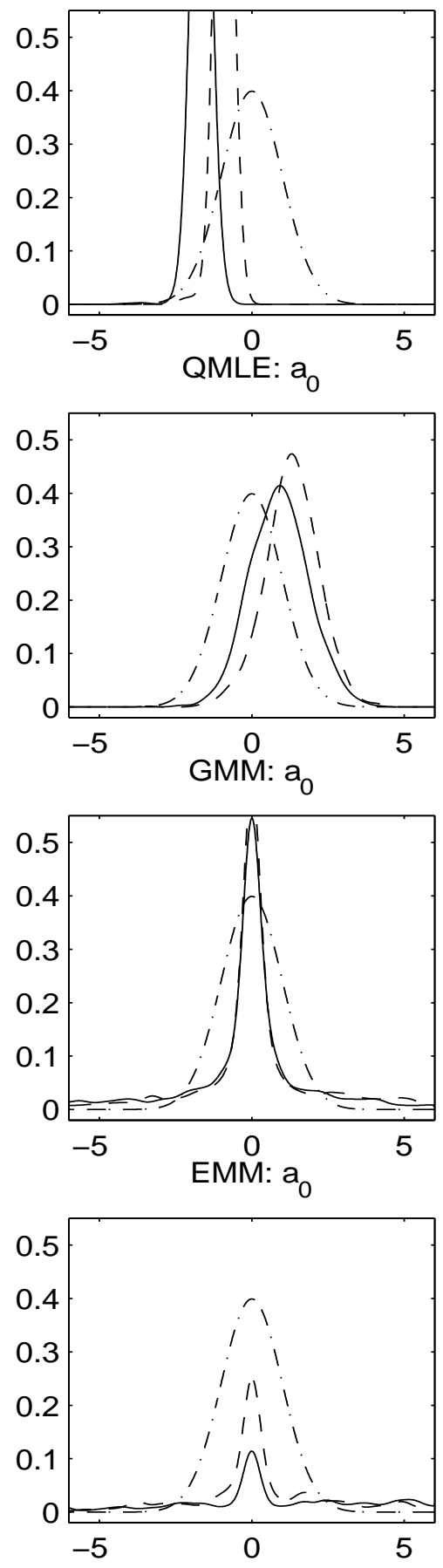

MLE: $a_{1}$
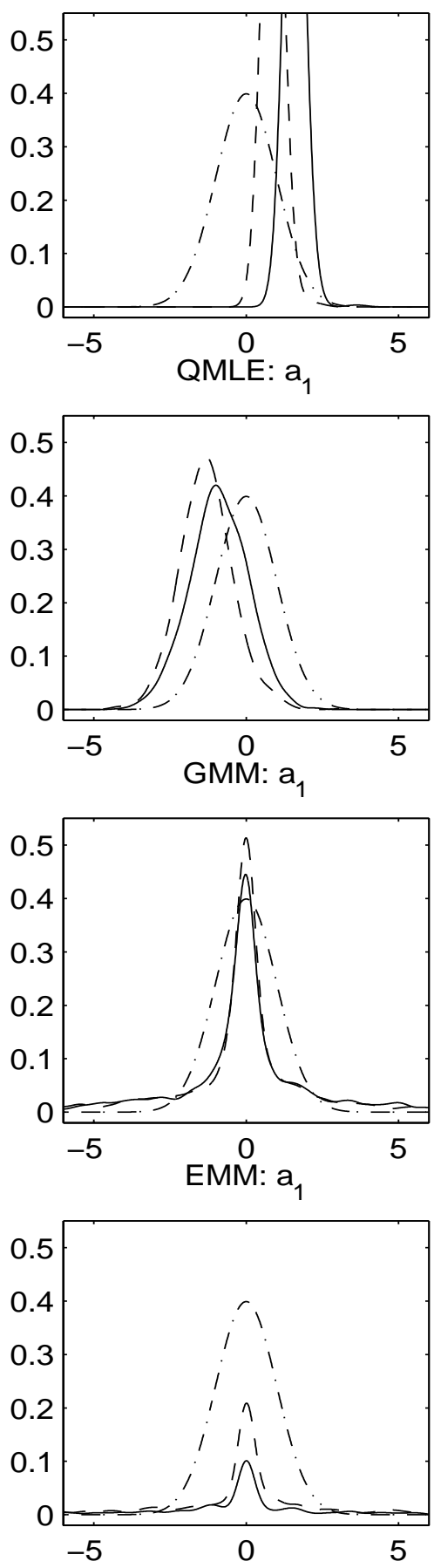

MLE: $b_{0}$
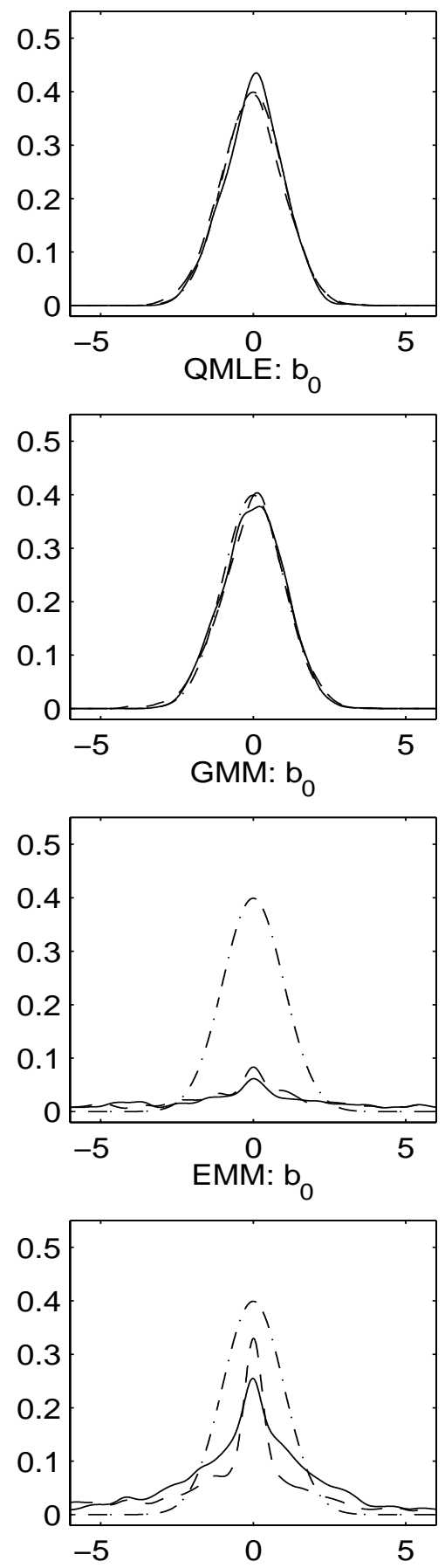

Figure 1: Sampling Distributions of t-Statistics for Scenario 1. The notations are respectively: “- - " t-test statistics for 500 sample size; "_-_ t-test statistics for 1500 sample size; “-.-.-” Normal $(0,1)$ density as the reference. 
MLE: $a_{0}$
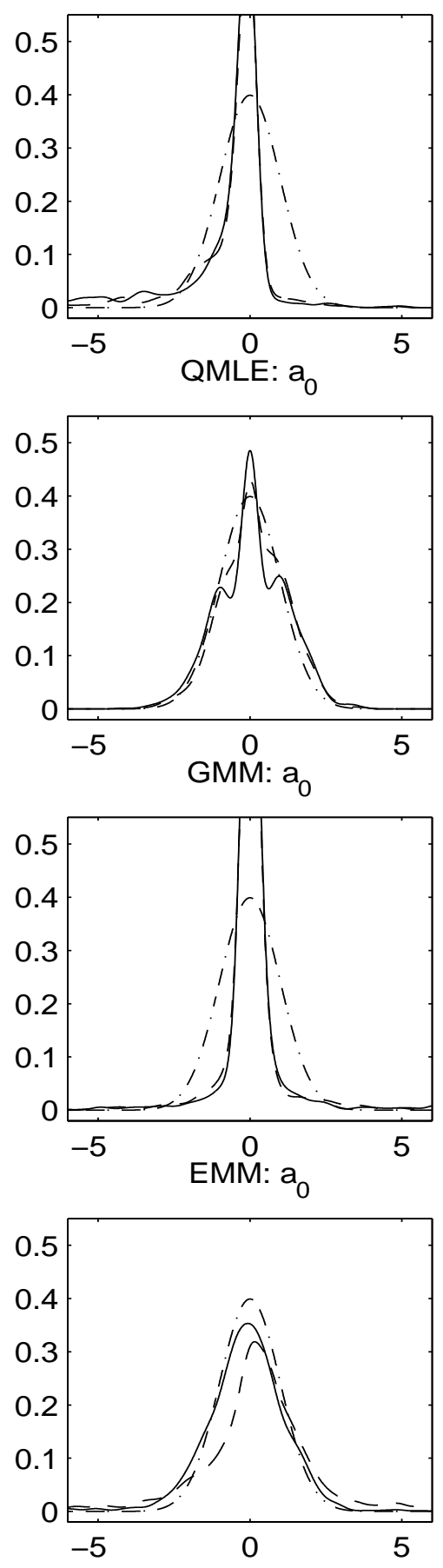

MLE: $\mathrm{a}_{1}$
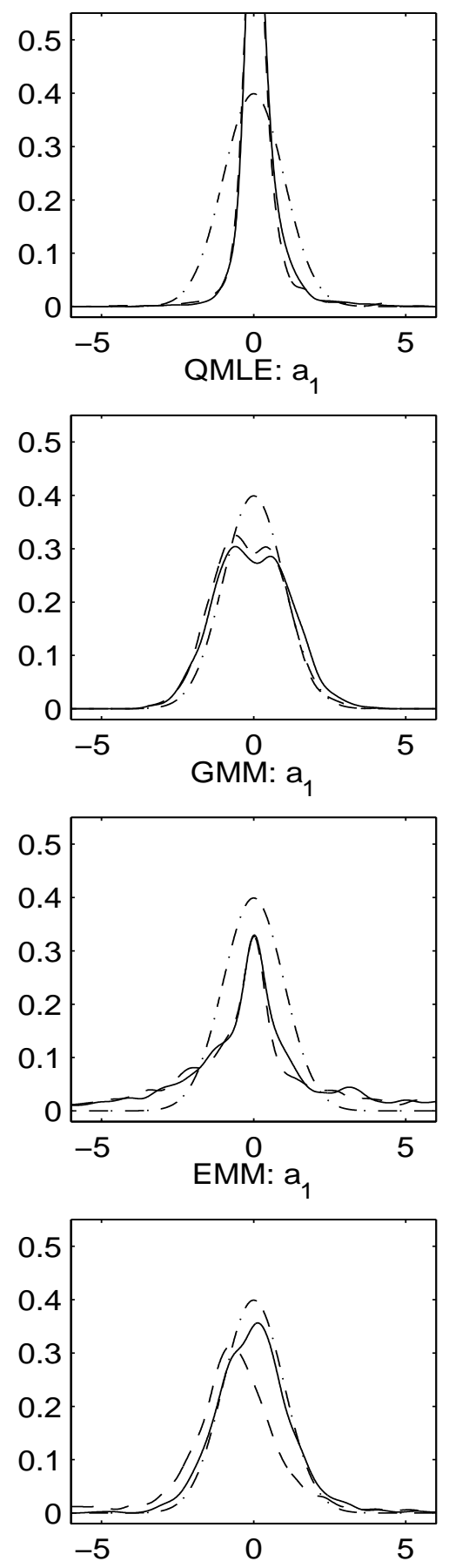

MLE: $b_{0}$
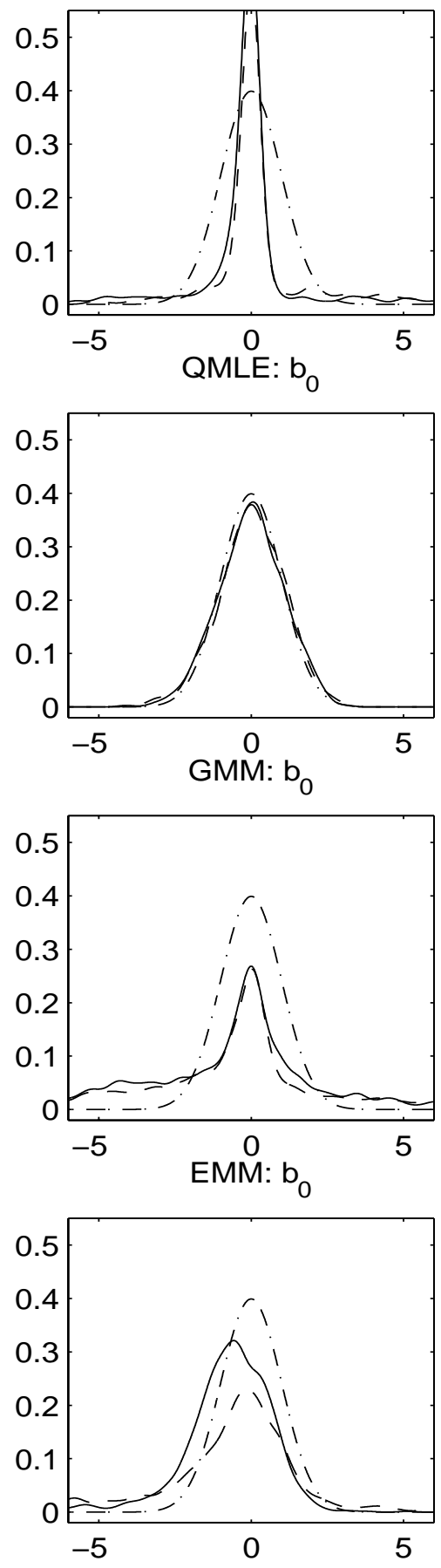

Figure 2: Sampling Distributions of t-Statistics for Scenario 8. The notations are respectively: “- - " t-test statistics for 500 sample size; "_-_ t-test statistics for 1500 sample size; “-.-.-" Normal $(0,1)$ density as the reference. 


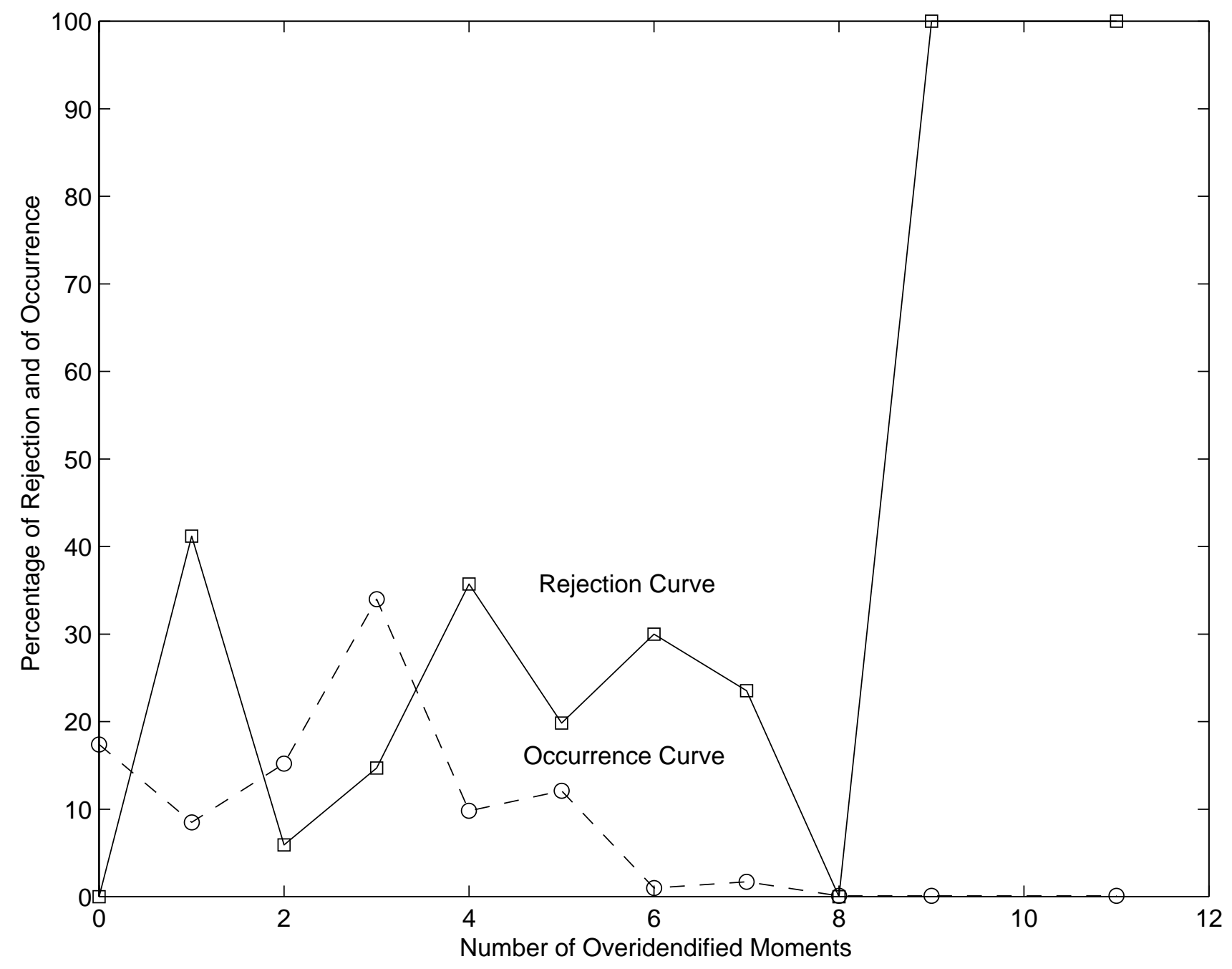

Figure 3: 5\% Overrejection Rate of EMM T $=500$ with Automatic Score Generator. The occurrence rate is the frequency of the same moment choice divided by 1000 . The rejection rate is the frequency of rejections divided by the number of occurrences. 


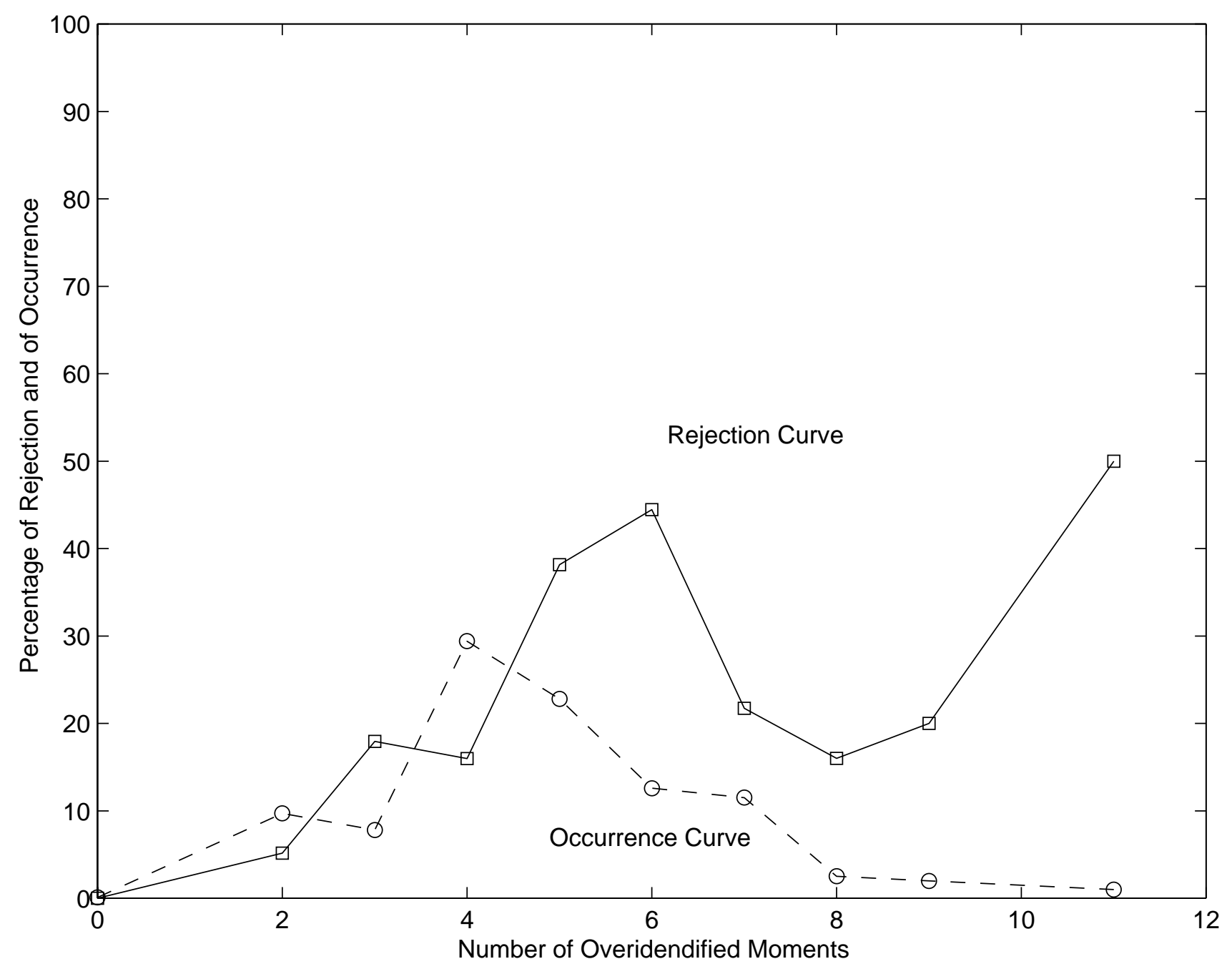

Figure 4: $5 \%$ Overrejection Rate of EMM T $=1500$ with Automatic Score Generator. The occurrence rate is the frequency of the same moment choice divided by 1000 . The rejection rate is the frequency of rejections divided by the number of occurrences. 

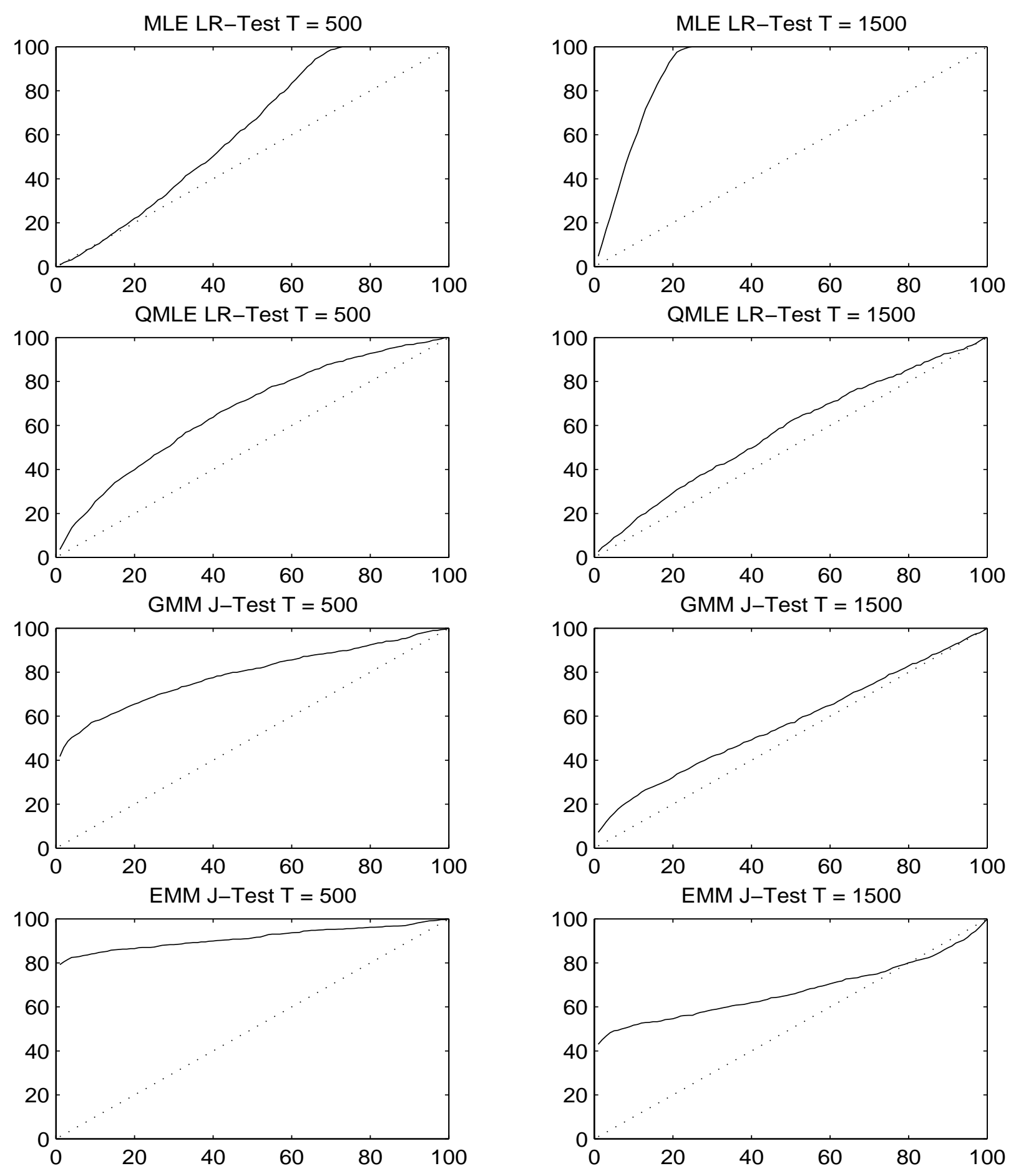

Figure 5: Specification Test for Scenario 1.

The likelihood ratio tests for MLE and QMLE are against the true parameter values, and the J-tests for GMM and EMM are against the overidentifying restrictions. 

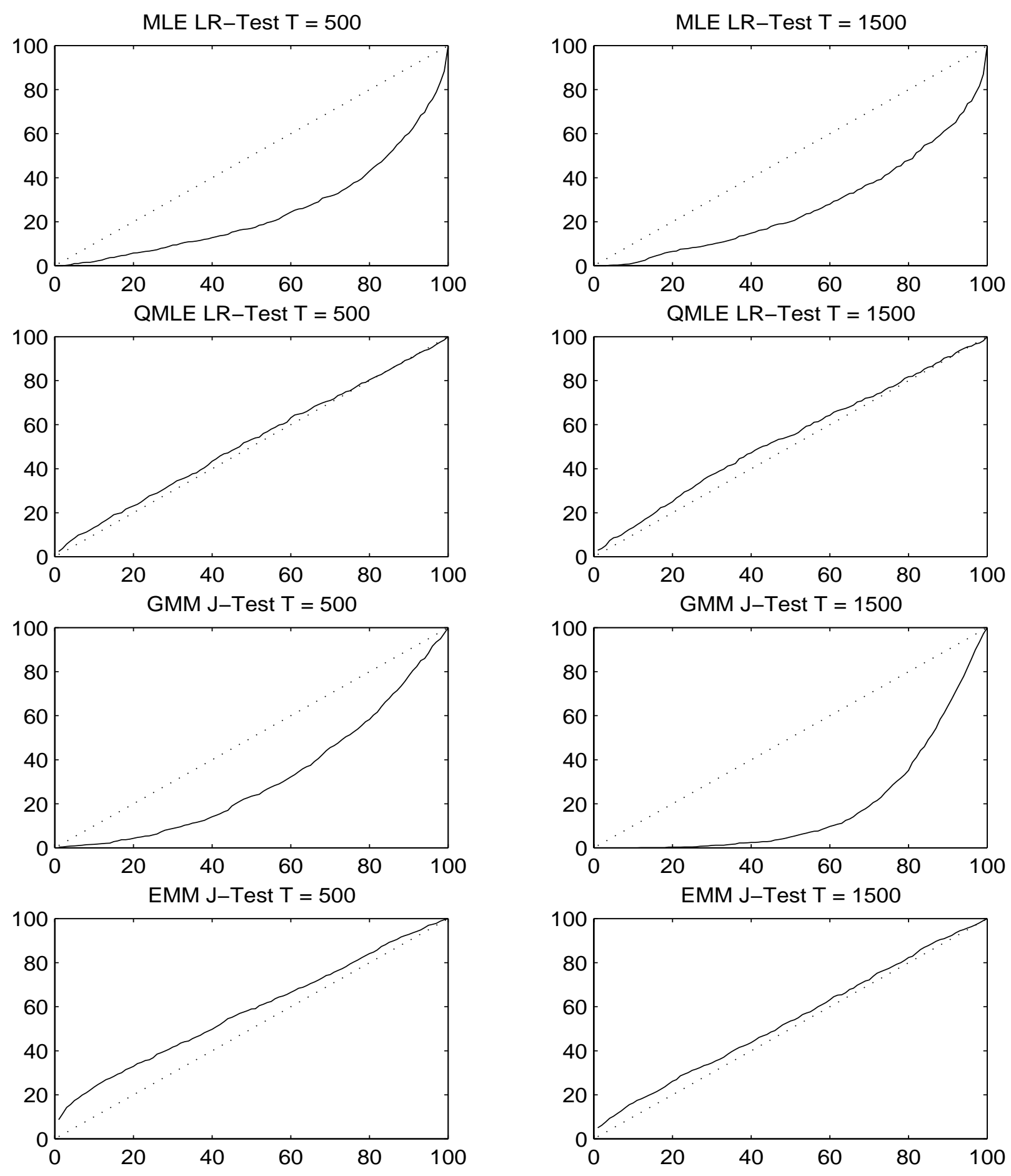

Figure 6: Specification Test for Scenario 8.

The likelihood ratio tests for MLE and QMLE are against the true parameter values, and the J-tests for GMM and EMM are against the overidentifying restrictions. 


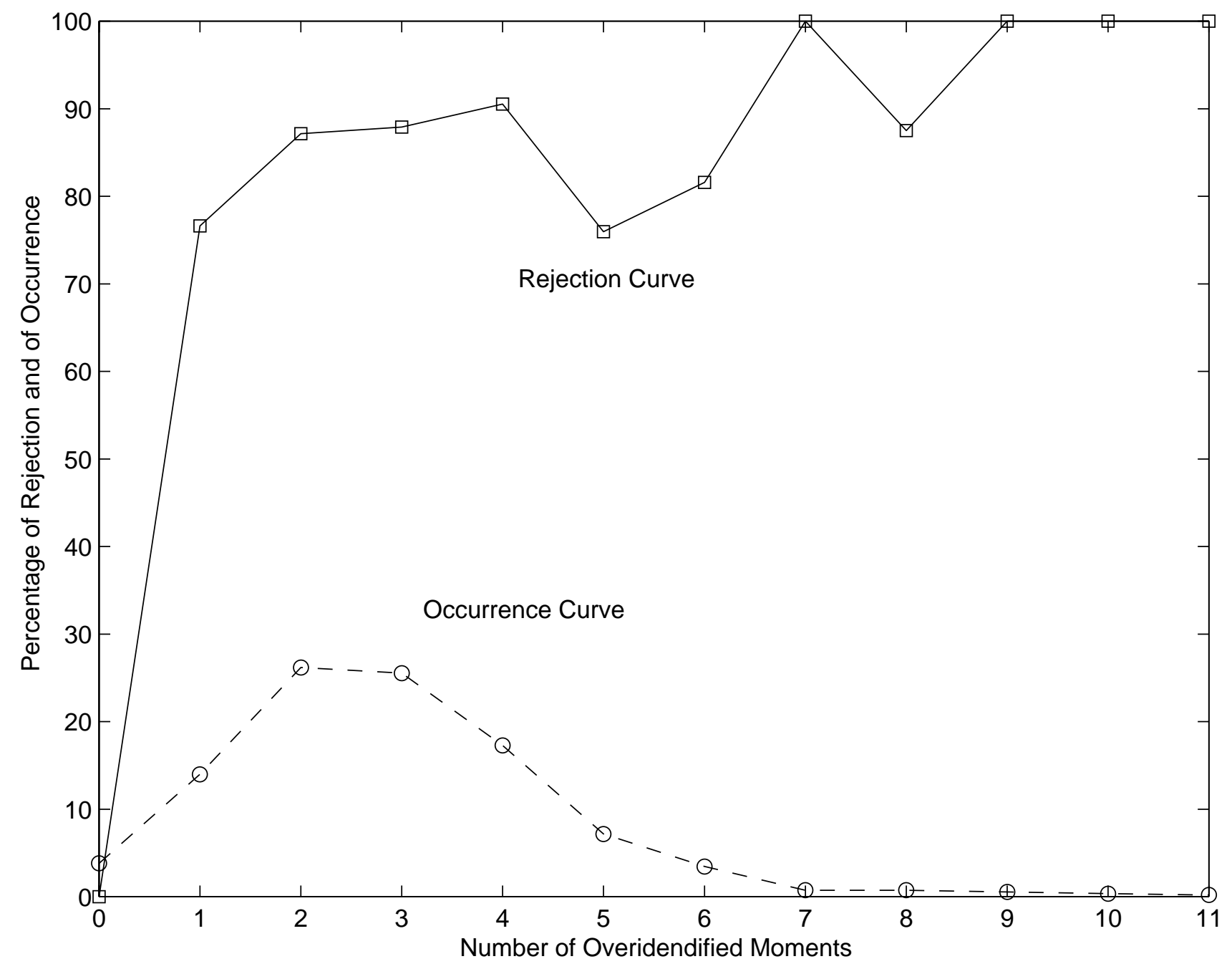

Figure 7: 5\% Rejection of Misspecified Model $\mathrm{T}=500$

The occurrence rate is the frequency of the same moment choice divided by 1000 . The rejection rate is the frequency of rejections divided by the number of occurrences. 


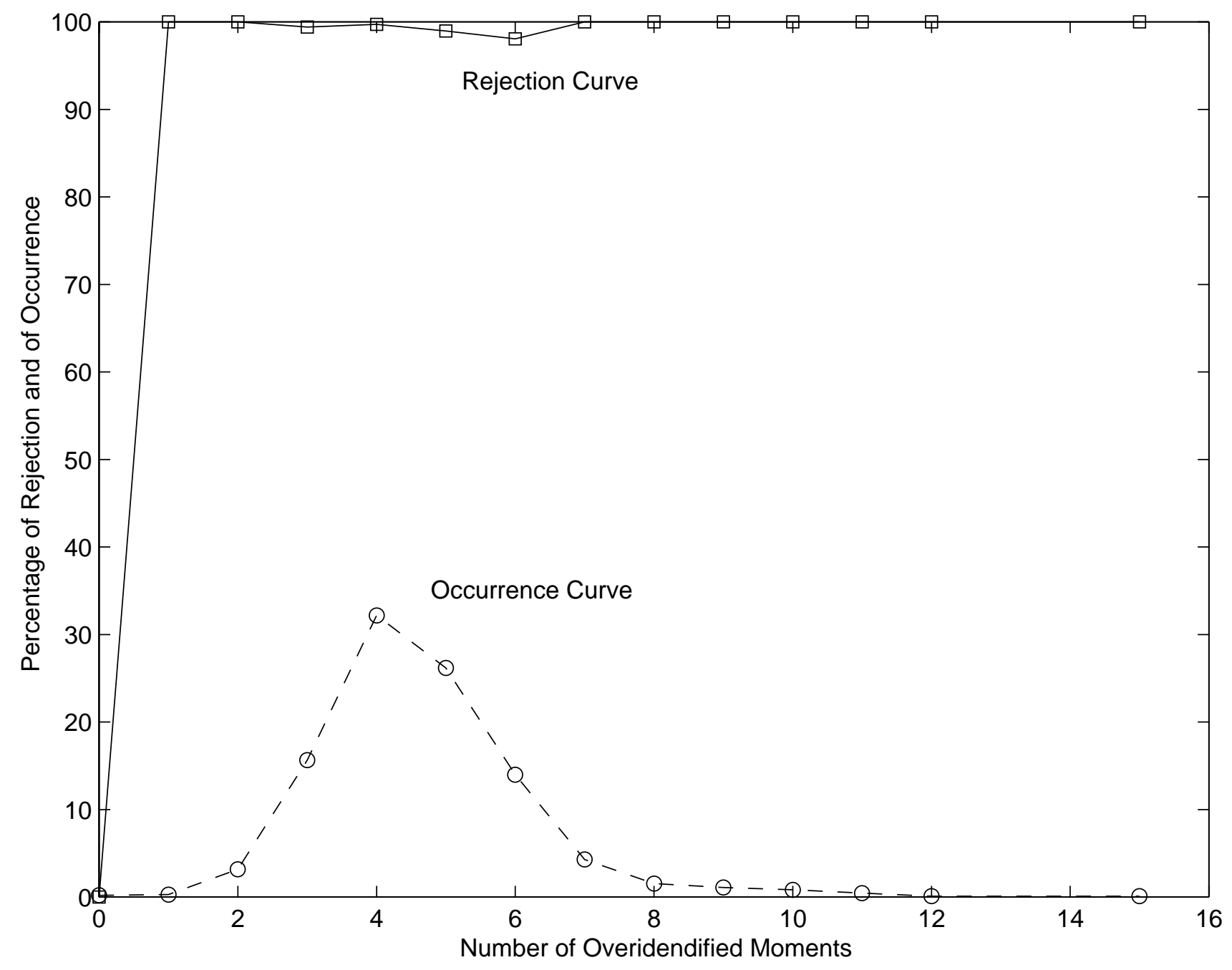

Figure 8: $5 \%$ Rejection of Misspecified Model $\mathrm{T}=1500$

The occurrence rate is the frequency of the same moment choice divided by 1000 . The rejection rate is the frequency of rejections divided by the number of occurrences. 

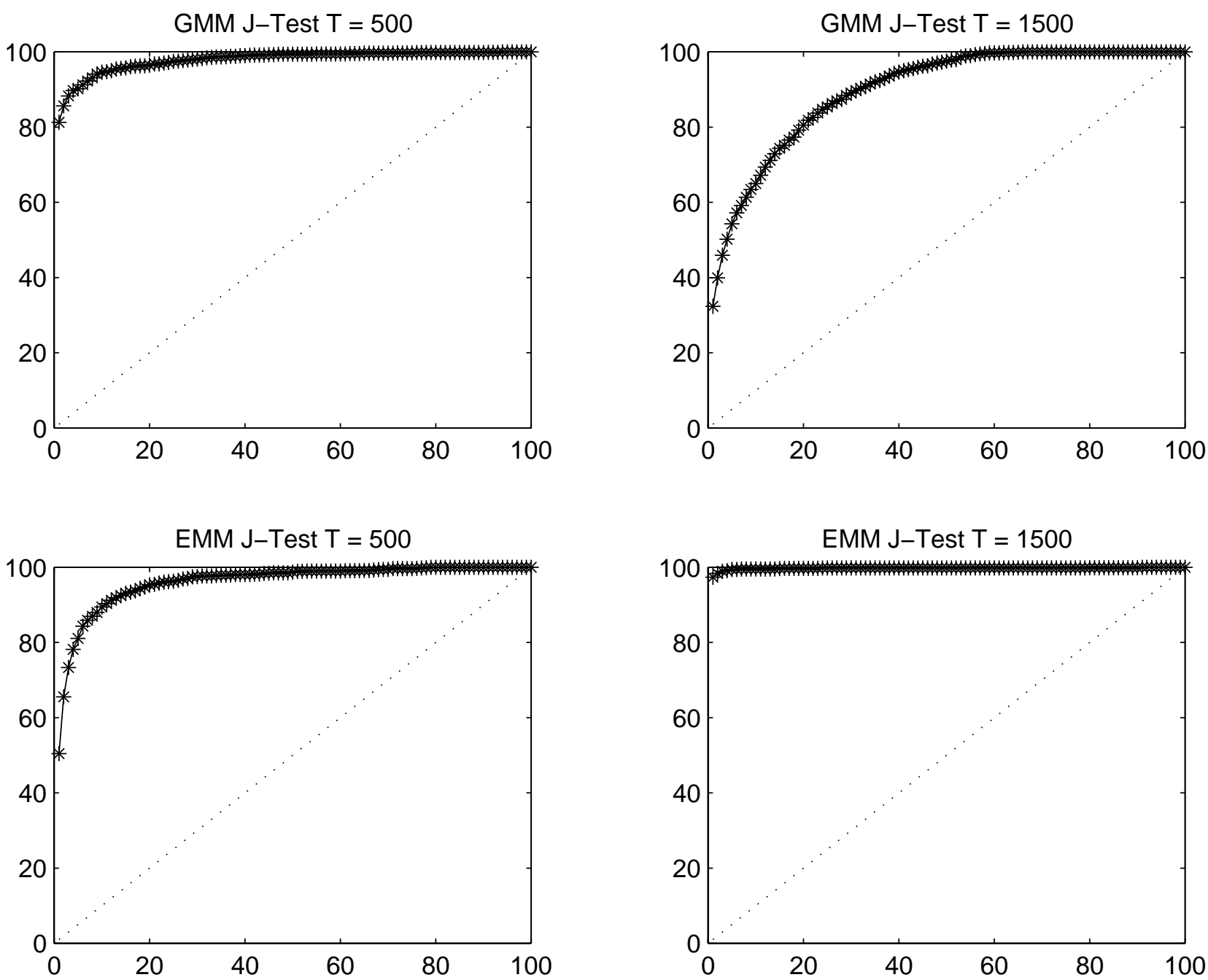

Figure 9: Power to Detect Misspecified Model.

The GMM J-test is a chi-square (1), and the EMM J-test is a chi-square (3) for $\mathrm{T}=500$ and chi-square (5) for $\mathrm{T}=1500$. 